\title{
On the Convergence of a Family of Chlodowsky Type Bernstein-Stancu-Schurer Operators
}

\author{
Lian-Ta Shu, ${ }^{1}$ Guorong Zhou, ${ }^{2}$ and Qing-Bo Cai ${ }^{1}{ }^{1}$ \\ ${ }^{1}$ School of Mathematics and Computer Science, Quanzhou Normal University, Quanzhou 362000, China \\ ${ }^{2}$ School of Applied Mathematics, Xiamen University of Technology, Xiamen 361024, China \\ Correspondence should be addressed to Qing-Bo Cai; qbcai@126.com
}

Received 26 April 2018; Revised 15 June 2018; Accepted 27 June 2018; Published 18 July 2018

Academic Editor: Gestur Ólafsson

Copyright (c) 2018 Lian-Ta Shu et al. This is an open access article distributed under the Creative Commons Attribution License, which permits unrestricted use, distribution, and reproduction in any medium, provided the original work is properly cited.

\begin{abstract}
We construct a new family of univariate Chlodowsky type Bernstein-Stancu-Schurer operators and bivariate tensor product form. We obtain the estimates of moments and central moments of these operators, obtain weighted approximation theorem, establish local approximation theorems by the usual and the second order modulus of continuity, estimate the rate of convergence, give a convergence theorem for the Lipschitz continuous functions, and also obtain a Voronovskaja-type asymptotic formula. For the bivariate case, we give the rate of convergence by using the weighted modulus of continuity. We also give some graphs and numerical examples to illustrate the convergent properties of these operators to certain functions and show that the new ones have a better approximation to functions $f$ for one dimension.
\end{abstract}

\section{Introduction}

In 1912, Bernstein [1] proposed the famous polynomials called nowadays Bernstein polynomials to prove the Weierstrass approximation theorem. Later it was found that Bernstein polynomials possess many remarkable properties, which made them an area of intensive research. Even recently, there are also many papers mentioned about Bernstein type operators, such as [2-5]. A generalization of Bernstein polynomials based on $q$-integers was proposed by Lupas in 1987 in [6]. However, the Lupas $q$-Bernstein operators are rational functions rather than polynomials. The Phillips $q$-Bernstein polynomials were introduced by Phillips in 1997 in [7]. In 2015, Mursaleen et al. [8] first introduced the $(p, q)$-analogue of Bernstein operators, in the case $p=1$ these operators coincide with the Phillips $q$-Bernstein operators. In the same year, they also proposed the $(p, q)$-analogue of BernsteinStancu operators in [9]. And then, in 2017, Khan et al. introduced the Lupas $(p, q)$-analogue of Bernstein operators. There are some recent papers relevant to Bernstein operators based on $(p, q)$-integers, such as [10-12]. Also some other positive operators related to $(p, q)$-integers; we listed some of them as [13-18].
In 1932, Chlodowsky introduced the classical BernsteinChlodowsky operators as

$$
\widetilde{B_{n}}(f ; x)=\sum_{k=0}^{n} f\left(\frac{k}{n} b_{n}\right)\left(\begin{array}{l}
n \\
k
\end{array}\right)\left(\frac{x}{b_{n}}\right)^{k}\left(1-\frac{x}{b_{n}}\right)^{n-k},
$$

where $0 \leq x \leq b_{n}$ and $\left\{b_{n}\right\}$ is a sequence of positive numbers such that $\lim _{n \rightarrow \infty} b_{n}=\infty, \lim _{n \rightarrow \infty}\left(b_{n} / n\right)=0$. These operators have been studied extensively, including one- and two-dimensional cases, which may be found in [19-25]. In 2017, Mishra et al. [26] introduced the Chlodowsky variant of $(p, q)$ Bernstein-Stancu-Schurer operators as

$$
\begin{aligned}
C_{n, m}^{(\alpha, \beta)}(f ; x, p, q) & =\frac{1}{p^{((n+m)(n+m-1)) / 2}} \\
& \cdot \sum_{k=0}^{n+m}\left[\begin{array}{c}
n+m \\
k
\end{array}\right]_{p, q} p^{k(k-1) / 2}\left(\frac{x}{b_{n}}\right)^{k} \\
& \cdot \prod_{s=0}^{n+m-k-1}\left(p^{s}-q^{s} \frac{x}{b_{n}}\right) \times f\left(\frac{p^{n+m-k}[k]_{p, q}+\alpha}{[n]_{p, q}+\beta} b_{n}\right),
\end{aligned}
$$

where $n \in \mathbb{N}, m, \alpha, \beta \in \mathbb{N}_{0}$, with $\alpha / \beta \approx 1,0 \leq x \leq b_{n}, 0<$ $q<p \leq 1$, and $b_{n}$ is an increasing sequence of positive terms 
with the properties $b_{n} \longrightarrow \infty$ and $b_{n} /[n]_{p, q} \longrightarrow 0$ as $n \longrightarrow \infty$. They discussed Korovkin-type approximation properties and rate of convergence of operators (2).

Due to the fact that these operators (2) reproduce only constant functions and it seems that there have been no twodimensional case of their defined operators (2) at present, the first aim of this paper is to give a new type of these operators such that the new ones preserve not only constant functions but also linear functions; the second aim is to introduce the two-dimensional case based on these operators (3), which will be defined in (69). We also discuss weighted approximation properties of these new operators (3) and (69) and compare with the ones (2) by graphics and the absolute error bound of numerical analysis; we will show that the new ones (3) are better than (2) when approximating to functions $f$.

We introduce new Chlodowsky type $(p, q)$-BernsteinStancu-Schurer operators $\widetilde{C_{n, p, q}^{\alpha, \beta, l}}(f ; x)$ as

$$
\begin{aligned}
& \widetilde{C_{n, p, q}^{\alpha, \beta, l}}(f ; x) \\
& \quad=\sum_{k=0}^{n+l} c_{n, p, q}^{k, l}(u(x)) f\left(\frac{p^{n+l-k}[k]_{p, q}+\alpha}{[n]_{p, q}+\beta} b_{n}\right),
\end{aligned}
$$

and the basis function $c_{n, p, q}^{k, l}(x)$ is defined as

$$
\begin{aligned}
c_{n, p, q}^{k, l}(x)= & \frac{1}{p^{(n+l)(n+l-1) / 2}}\left[\begin{array}{c}
n+l \\
k
\end{array}\right]_{p, q} p^{k(k-1) / 2}\left(\frac{x}{b_{n}}\right)^{k} \\
& \cdot\left(1-\frac{x}{b_{n}}\right)_{p, q}^{n+l-k},
\end{aligned}
$$

where $0<q<p \leq 1, u(x)=\left(\left(\left([n]_{p, q}+\beta\right) x-\alpha b_{n}\right) /[n+\right.$ $\left.l]_{p, q}\right)\left(\alpha b_{n} /\left([n]_{p, q}+\beta\right) \leq x \leq\left(\left([n+l]_{p, q}+\alpha\right) /\left([n]_{p, q}+\beta\right)\right) b_{n}\right)$, $0 \leq \alpha \leq \beta$, and $b_{n}$ is an increasing sequence of positive terms with the properties $b_{n} \longrightarrow \infty, b_{n} /[n]_{p, q} \longrightarrow 0$ as $n \longrightarrow \infty$.

We mention some definitions based on $(p, q)$-integers, and details can be found in [27-31]. For any fixed real number $p>0$ and $q>0$, the $(p, q)$-integers $[k]_{p, q}$ are defined by

$$
\begin{aligned}
{[k]_{p, q} } & =p^{k-1}+p^{k-2} q+p^{k-3} q^{2}+\cdots+p q^{k-2}+q^{k-1} \\
& = \begin{cases}\frac{p^{k}-q^{k}}{p-q}, & p \neq q \neq 1 ; \\
k p^{k-1}, & p=q \neq 1 ; \\
{[k]_{q},} & p=1 ; \\
k, & p=q=1,\end{cases}
\end{aligned}
$$

where $[k]_{q}$ denotes the $q$-integers and $k=0,1,2, \ldots$ Also $(p, q)$-factorial and $(p, q)$-binomial coefficients are defined as follows:

$$
[k]_{p, q} != \begin{cases}{[k]_{p, q}[k-1]_{p, q} \cdots[1]_{p, q},} & k=1,2, \ldots, \\ 1, & k=0,\end{cases}
$$

$$
\left[\begin{array}{l}
n \\
k
\end{array}\right]_{p, q}=\frac{[n]_{p, q} !}{[k]_{p, q} ![n-k]_{p, q} !}, \quad(n \geq k \geq 0) .
$$

The $(p, q)$-Binomial expansion is defined by

$$
\begin{aligned}
& (x+y)_{p, q}^{n} \\
& = \begin{cases}1, & n=0, \\
(x+y)(p x+q y) \cdots\left(p^{n-1} x+q^{n-1} y\right), & n=1,2, \ldots\end{cases}
\end{aligned}
$$

When $p=1$, all the definitions of $(p, q)$-calculus above are reduced to $q$-calculus.

The paper is organized as follows: In Section 2, we give some basic definitions regarding $(p, q)$-integers. In Section 3, we estimate the moments and central moments of these operators (3). In Section 4, we obtain weighted approximation theorem, establish local approximation theorems, estimate the rate of convergence, give a convergence theorem for the Lipschitz continuous functions, and also obtain a Voronovskaja-type asymptotic formula. In Section 5, we give some graphs and numerical examples to illustrate the convergent properties for one variable functions and compare with the ones in (2). In Section 6, we propose the bivariate case, give the rate of convergence by using the weighted modulus of continuity, and give some graphs and numerical analysis for two variables functions.

\section{Auxiliary Results}

Lemma 1 (see [26], Lemma 2). For $x \in\left[0, b_{n}\right]$, we have

$$
\begin{aligned}
& C_{n, m}^{(\alpha, \beta)}(1 ; x, p, q)=1, \\
& C_{n, m}^{(\alpha, \beta)}(t ; x, p, q)=\frac{[n+m]_{p, q} x+\alpha b_{n}}{[n]_{p, q}+\beta}, \\
& C_{n, m}^{(\alpha, \beta)}\left(t^{2} ; x, p, q\right)=\frac{q[n+m]_{p, q}[n+m-1]_{p, q}}{\left([n]_{p, q}+\beta\right)^{2}} x^{2} \\
& \quad+\frac{[n+m]_{p, q}\left(2 \alpha+p^{n+m-1}\right) b_{n}}{\left([n]_{p, q}+\beta\right)^{2}} x+\frac{\alpha^{2} b_{n}^{2}}{\left([n]_{p, q}+\beta\right)^{2}}, \\
& C_{n, m}^{(\alpha, \beta)}(t-x ; x, p, q)=\left(\frac{[n+m]_{p, q}}{[n]_{p, q}+\beta}-1\right) x \\
& +\frac{\alpha b_{n}}{[n]_{p, q}+\beta}, \\
& C_{n, m}^{(\alpha, \beta)}\left((t-x)^{2} ; x, p, q\right)=\left(1-2 \frac{[n+m]_{p, q}}{[n]_{p, q}+\beta}\right. \\
& \left.\quad+\frac{q[n+m]_{p, q}[n+m-1]_{p, q}}{\left([n]_{p, q}+\beta\right)^{2}}\right) x^{2}
\end{aligned}
$$




$$
\begin{aligned}
& +\left(\frac{\left(2 \alpha+p^{n+m-1}\right)[n+m]_{p, q}}{[n]_{p, q}+\beta}-2 \alpha\right) \frac{b_{n} x}{[n]_{p, q}+\beta} \\
& +\frac{\alpha^{2} b_{n}^{2}}{\left([n]_{p, q}+\beta\right)^{2}} .
\end{aligned}
$$

Lemma 2. For $x \in\left[\alpha b_{n} /\left([n]_{p, q}+\beta\right),\left(\left([n+l]_{p, q}+\alpha\right) /\left([n]_{p, q}+\right.\right.\right.$ $\left.\beta)) b_{n}\right]$, the following equalities hold:

$$
\begin{aligned}
\widetilde{C_{n, p, q}^{\alpha, \beta, l}}(1 ; x)= & 1, \\
\widetilde{C_{n, p, q}^{\alpha, \beta, l}}(t ; x)= & x, \\
\widetilde{C_{n, p, q}^{\alpha, \beta, l}}\left(t^{2} ; x\right)= & x^{2}-\frac{p^{n+l-1} x^{2}}{[n+l]_{p, q}} \\
& +\frac{p^{n+l-1} b_{n}\left(2 \alpha+[n+l]_{p, q}\right) x}{\left([n]_{p, q}+\beta\right)[n+l]_{p, q}} \\
\widetilde{C_{n, p, q}^{\alpha, \beta, l}}(t-x ; x)= & -\frac{p^{n+l-1} \alpha b_{n}^{2}\left(\alpha+[n+l]_{p, q}\right)}{\left([n]_{p, q}+\beta\right)^{2}[n+l]_{p, q}}, \\
\widetilde{C_{n, p, q}^{\alpha, \beta, l}}\left((t-x)^{2} ; x\right)= & \frac{p^{n+l-1} b_{n}\left(2 \alpha+[n+l]_{p, q}\right) x}{\left([n]_{p, q}+\beta\right)[n+l]_{p, q}} \\
& -\frac{p^{n+l-1} \alpha b_{n}^{2}\left(\alpha+[n+l]_{p, q}\right)}{\left([n]_{p, q}+\beta\right)^{2}[n+l]_{p, q}} \\
& -\frac{p^{n+l-1} x^{2}}{[n+l]_{p, q}} .
\end{aligned}
$$

Proof. From Lemma 1 and (3), we obtain (9), (10), and (11) by some simple computations, and we also get (12) and (13) by (9)-(11); here we omit it.

Remark 3. From (9) and (10) of Lemma 2, we know the operators $\widetilde{C_{n, p, q}^{\alpha, \beta, l}}(f ; x)$ preserve not only constant functions but also linear functions. That is to say, $\widetilde{C_{n, p, q}^{\alpha, \beta, l}}(a t+b ; x)=$ $a x+b$, where fixed $a, b \in \mathbb{R}$.

Lemma 4 (see Theorem 2.1 of [32]). For $0<q_{n}<p_{n} \leq 1$, set $q_{n}:=1-\alpha_{n}, p_{n}:=1-\beta_{n}$ such that $0 \leq \beta_{n}<\alpha_{n}<1, \alpha_{n} \longrightarrow 0$, $\beta_{n} \longrightarrow 0$ as $n \longrightarrow \infty$. The following statements are true:

\footnotetext{
(A) If $\lim _{n \rightarrow \infty} e^{n\left(\beta_{n}-\alpha_{n}\right)}=1$ and $e^{n \beta_{n}} / n \longrightarrow 0$, then $[n]_{p_{n}, q_{n}} \longrightarrow \infty$.

(B) If $\overline{\lim }_{n \rightarrow \infty} e^{n\left(\beta_{n}-\alpha_{n}\right)}<1$ and $e^{n \beta_{n}}\left(\alpha_{n}-\beta_{n}\right) \longrightarrow 0$, then $[n]_{p_{n}, q_{n}} \longrightarrow \infty$.

(C) If $\underline{\lim }_{n \rightarrow \infty} e^{n\left(\beta_{n}-\alpha_{n}\right)}<1, \varlimsup_{n \rightarrow \infty} e^{n\left(\beta_{n}-\alpha_{n}\right)}=1$ and $\max \left\{e^{n \beta_{n}} / n, e^{n \beta_{n}}\left(\alpha_{n}-\beta_{n}\right)\right\} \longrightarrow 0$, then $[n]_{p_{n}, q_{n}} \longrightarrow \infty$.
}

Lemma 5. Consider the sequences $p=\left\{p_{n}\right\}$ and $q=\left\{q_{n}\right\}$, for $0<q_{n}<p_{n} \leq 1$ satisfying the conditions $(A),(B)$, or $(C)$ of Lemma 4 and $\lim _{n \rightarrow \infty} p_{n}^{n}=a, a \in(0,1]$; then for fixed $x \in\left[\alpha b_{n} /\left([n]_{p, q}+\beta\right),\left(\left([n+l]_{p, q}+\alpha\right) /\left([n]_{p, q}+\beta\right)\right) b_{n}\right]$, the following equalities hold:

$$
\begin{aligned}
& \widetilde{C_{n, p, q}^{\alpha, \beta, l}}\left(t^{3} ; x\right)=x^{3}+O\left(\frac{b_{n}}{[n]_{p, q}}\right) \varphi_{1}(x), \\
& \widetilde{C_{n, p, q}^{\alpha, \beta, l}}\left(t^{4} ; x\right)=x^{4}+O\left(\frac{b_{n}}{[n]_{p, q}}\right) \varphi_{2}(x), \\
& \lim _{n \longrightarrow \infty} \frac{[n]_{p_{n}, q_{n}}}{b_{n}} \widetilde{C_{n, p_{n}, q_{n}}^{\alpha, \beta, l}}\left((t-x)^{2} ; x\right)=a x, \\
& \lim _{n \longrightarrow \infty} \frac{[n]_{p_{n}, q_{n}}^{2}}{b_{n}^{2}} \widetilde{C_{n, p_{n}, q_{n}}^{\alpha, \beta,}}\left((t-x)^{4} ; x\right)=3 a^{2} x^{2},
\end{aligned}
$$

where $\varphi_{1}(x)$ and $\varphi_{2}(x)$ are functions depending on $x$.

Proof. From (3), we have

$$
\begin{gathered}
\widetilde{C_{n, p, q}^{\alpha, \beta, l}}\left(t^{3} ; x\right)=\sum_{k=0}^{n+l} c_{n, p, q}^{k, l}(x)\left(\frac{p^{n+l-k}[k]_{p, q}+\alpha}{[n]_{p, q}+\beta} b_{n}\right)^{3} \\
=\sum_{k=0}^{n+l} c_{n, p, q}^{k, l}(x)\left(\frac{p^{3 n+3 l-3 k}[k]_{p, q}^{3} b_{n}^{3}}{\left([n]_{p, q}+\beta\right)^{3}}\right. \\
+\frac{3 p^{2 n+2 l-2 k}[k]_{p, q}^{2} \alpha b_{n}^{3}}{\left([n]_{p, q}+\beta\right)^{3}}+\frac{3 p^{n+l-k}[k]_{p, q} \alpha^{2} b_{n}^{3}}{\left([n]_{p, q}+\beta\right)^{3}} \\
\left.+\frac{\alpha^{3} b_{n}^{3}}{\left([n]_{p, q}+\beta\right)^{3}}\right),:=\triangle_{1}+\triangle_{2}+\triangle_{3}+\triangle_{4},
\end{gathered}
$$

and by some computations, we have

$$
\begin{aligned}
{[k]_{p, q}^{2}=} & p^{k-1}[k]_{p, q}+q[k]_{p, q}[k-1]_{p, q}, \\
{[k]_{p, q}^{3}=} & q^{3}[k]_{p, q}[k-1]_{p, q}[k-2]_{p, q} \\
& +p^{k-2} q(2 p+q)[k]_{p, q}[k-1]_{p, q} \\
& +p^{2 k-2}[k]_{p, q}
\end{aligned}
$$

thus, using (4), (19), (20), and $\sum_{k=0}^{n+l} c_{n, p, q}^{k, l}(x)=1$, we have

$$
\begin{aligned}
\triangle_{1} & =\sum_{k=0}^{n+l} c_{n, p, q}^{k, l}(x) \frac{p^{3 n+3 l-3 k}[k]_{p, q}^{3} b_{n}^{3}}{\left([n]_{p, q}+\beta\right)^{3}} \\
& =\frac{q^{3}[n+l]_{p, q}[n+l-1]_{p, q}[n+l-2]_{p, q}}{\left([n]_{p, q}+\beta\right)^{3}}(u(x))^{3}
\end{aligned}
$$




$$
\begin{aligned}
& +\frac{p^{n+l-2} q(2 p+q) b_{n}[n+l]_{p, q}[n+l-1]_{p, q}}{\left([n]_{p, q}+\beta\right)^{3}}(u(x))^{2} \\
& +\frac{p^{2 n+2 l-2} b_{n}^{2}[n+l]_{p, q}}{\left([n]_{p, q}+\beta\right)^{3}} u(x),
\end{aligned}
$$

and, similarly, we have

$$
\begin{aligned}
\triangle_{2}= & \sum_{k=0}^{n+l} c_{n, p, q}^{k, l}(x) \frac{3 p^{2 n+2 l-2 k}[k]_{p, q}^{2} \alpha b_{n}^{3}}{\left([n]_{p, q}+\beta\right)^{3}} \\
= & \frac{3 q \alpha b_{n}[n+l]_{p, q}[n+l-1]_{p, q}}{\left([n]_{p, q}+\beta\right)^{3}}(u(x))^{2} \\
& +\frac{3 p^{n+l-1} \alpha b_{n}^{2}[n+l]_{p, q}}{\left([n]_{p, q}+\beta\right)^{3}} u(x), \\
\triangle_{3}= & \sum_{k=0}^{n+l} c_{n, p, q}^{k, l}(x) \frac{3 p^{n+l-k}[k]_{p, q} \alpha^{2} b_{n}^{3}}{\left([n]_{p, q}+\beta\right)^{3}} \\
= & \frac{3 \alpha^{2} b_{n}^{2}[n+l]_{p, q} u(x),}{\left([n]_{p, q}+\beta\right)^{3}}, \\
\triangle_{4}= & \sum_{k=0}^{n+l} c_{n, p, q}^{k, l}(x) \frac{\alpha^{3} b_{n}^{3}}{\left([n]_{p, q}+\beta\right)^{3}}=\frac{\alpha^{3} b_{n}^{3}}{\left([n]_{p, q}+\beta\right)^{3}} .
\end{aligned}
$$

Since $u(x)=\left(\left([n]_{p, q}+\beta\right) x-\alpha b_{n}\right) /[n+l]_{p, q}$, from (21), we get

$$
\begin{gathered}
\frac{q^{3}[n+l]_{p, q}[n+l-1]_{p, q}[n+l-2]_{p, q}}{\left([n]_{p, q}+\beta\right)^{3}}(u(x))^{3} \\
=\frac{q^{3}[n+l-1]_{p, q}[n+l-2]_{p, q}}{[n+l]_{p, q}^{2}} x^{3}
\end{gathered}
$$

$$
\begin{aligned}
& -\frac{3 \alpha b_{n} q^{3}[n+l-1]_{p, q}[n+l-2]_{p, q}}{\left([n]_{p, q}+\beta\right)[n+l]_{p, q}^{2}} x^{2} \\
& +\frac{3 \alpha^{2} b_{n}^{2} q^{3}[n+l-1]_{p, q}[n+l-2]_{p, q}}{\left([n]_{p, q}+\beta\right)^{2}[n+l]_{p, q}^{2}} x \\
& -\frac{\alpha^{3} b_{n}^{3} q^{3}[n+l-1]_{p, q}[n+l-2]_{p, q}}{\left([n]_{p, q}+\beta\right)^{3}[n+l]_{p, q}^{2}},
\end{aligned}
$$

due to

$$
\begin{aligned}
& \frac{q^{3}[n+l-1]_{p, q}[n+l-2]_{p, q}}{[n+l]_{p, q}^{2}} x^{3} \\
& =\frac{\left([n+l]_{p, q}-p^{n+l-1}\right)\left([n+l]_{p, q}-[2]_{p, q} p^{n+l-2}\right)}{[n+l]_{p, q}^{2}} x^{3},
\end{aligned}
$$

and, combining (18), (21)-(24), $u(x)=\left(\left([n]_{p, q}+\beta\right) x-\right.$ $\left.\alpha b_{n}\right) /[n+l]_{p, q},(25)$, and (26), we obtain

$$
\widetilde{C_{n, p, q}^{\alpha, \beta, l}}\left(t^{3} ; x\right)=x^{3}+O\left(\frac{b_{n}}{[n]_{p, q}}\right) \varphi_{1}(x) .
$$

Next, since

$$
\begin{aligned}
& {[k]_{p, q}^{4} } \\
&=q^{6}[k]_{p, q}[k-1]_{p, q}[k-2]_{p, q}[k-3]_{p, q} \\
&+p^{k-3} q^{3}\left([2]_{p, q}^{2}+2 p^{2}\right)[k]_{p, q}[k-1]_{p, q}[k-2]_{p, q} \\
&+p^{2 k-4} q\left(2 p[2]_{p, q}+[3]_{p, q}\right)[k]_{p, q}[k-1]_{p, q} \\
&+p^{3 k-3}[k]_{p, q},
\end{aligned}
$$

using the same methods and by some computations, we get

$$
\begin{aligned}
\widetilde{C_{n, p, q}^{\alpha, \beta, l}}\left(t^{4} ; x\right)= & \sum_{k=0}^{n+l} c_{n, p, q}^{k, l}(x)\left(\frac{p^{n+l-k}[k]_{p, q}+\alpha}{[n]_{p, q}+\beta} b_{n}\right)^{4} \\
= & \frac{q^{6}[n+l]_{p, q}[n+l-1]_{p, q}[n+l-2]_{p, q}[n+l-3]_{p, q}}{\left([n]_{p, q}+\beta\right)^{4}}(u(x))^{4} \\
& +\frac{p^{n+l-3} q^{3}\left([2]_{p, q}^{2}+2 p^{2}\right) b_{n}[n+l]_{p, q}[n+l-1]_{p, q}[n+l-2]_{p, q}}{\left([n]_{p, q}+\beta\right)^{4}}(u(x))^{3} \\
& +\frac{p^{2 n+2 l-4} q\left(2 p[2]_{p, q}+[3]_{p, q}\right) b_{n}^{2}[n+l]_{p, q}[n+l-1]_{p, q}}{\left([n]_{p, q}+\beta\right)^{4}}(u(x))^{2}+\frac{p^{3 n+3 l-3} b_{n}^{3}[n+l]_{p, q}}{\left([n]_{p, q}+\beta\right)^{4}} u(x)
\end{aligned}
$$




$$
\begin{aligned}
& +\frac{4 q^{3} \alpha b_{n}[n+l]_{p, q}[n+l-1]_{p, q}[n+l-2]_{p, q}}{\left([n]_{p, q}+\beta\right)^{4}}(u(x))^{3} \\
& +\frac{4 p^{n+l-2} q(2 p+q) \alpha b_{n}^{2}[n+l]_{p, q}[n+l-1]_{p, q}}{\left([n]_{p, q}+\beta\right)^{4}}(u(x))^{2}+\frac{4 p^{2 n+2 l-2} \alpha b_{n}^{3}[n+l]_{p, q}}{\left([n]_{p, q}+\beta\right)^{4}} u(x) \\
& +\frac{6 p^{n+l-1} \alpha^{2} b_{n}^{3}[n+l]_{p, q}}{\left([n]_{p, q}+\beta\right)^{4}} u(x)+\frac{6 q \alpha^{2} b_{n}^{2}[n+l]_{p, q}[n+l-1]_{p, q}}{\left([n]_{p, q}+\beta\right)^{4}}(u(x))^{2}+\frac{4 \alpha^{3} b_{n}^{3}[n+l]_{p, q}}{\left([n]_{p, q}+\beta\right)^{4}} u(x) \\
& +\frac{\alpha^{4} b_{n}^{4}}{\left([n]_{p, q}+\beta\right)^{4}},
\end{aligned}
$$

and, by formula (29), $u(x)=\left(\left([n]_{p, q}+\beta\right) x-\alpha b_{n}\right) /[n+l]_{p, q}$, and computations, we also have

$$
\widetilde{C_{n, p, q}^{\alpha, \beta, l}}\left(t^{4} ; x\right)=x^{4}+O\left(\frac{b_{n}}{[n]_{p, q}}\right) \varphi_{2}(x) .
$$

Equation (16) can easily be obtained by (13). Finally, using the above conclusions and computations, we get

$$
\begin{aligned}
\lim _{n \rightarrow \infty} & \frac{[n]_{p_{n}, q_{n}}^{2}}{b_{n}^{2}} \widetilde{C_{n, p_{n}, q_{n}}^{\alpha, \beta, l}}\left((t-x)^{4} ; x\right) \\
& =\lim _{n \rightarrow \infty} \frac{[n]_{p_{n}, q_{n}}^{2}}{b_{n}^{2}}\left(\widetilde{C_{n, p_{n}, q_{n}}^{\alpha, \beta, l}}\left(t^{4} ; x\right)-4 x \widetilde{C_{n, p_{n}, q_{n}}^{\alpha, \beta, l}}\left(t^{3} ; x\right)\right. \\
& \left.+6 x^{2} \widetilde{C_{n, p_{n}, q_{n}}^{\alpha, \beta, l}}\left(t^{2} ; x\right)-3 x^{4}\right)=3 a^{2} x^{2} .
\end{aligned}
$$

Lemma 5 is proved.

\section{Approximation Properties}

In the sequel, let $I=\left[\alpha b_{n} /\left([n]_{p, q}+\beta\right),\left(\left([n+l]_{p, q}+\right.\right.\right.$ $\left.\left.\alpha) /\left([n]_{p, q}+\beta\right)\right) b_{n}\right]$, where $0 \leq \alpha \leq \beta$ and $b_{n}$ is an increasing sequence of positive terms with $\lim _{n \rightarrow \infty} b_{n}=$ $\infty$ and $\lim _{n \rightarrow \infty}\left(b_{n} /[n]_{p, q}\right)=0$. In order to obtain the weighted approximation Theorem 6 , let $p=\left\{p_{n}\right\}, q=\left\{q_{n}\right\}$ be sequences satisfying the conditions $(A),(B)$, or $(C)$ of Lemma 4 and $\lim _{n \rightarrow \infty} p_{n}^{n}=a, a \in(0,1]$.

Let $B_{x^{2}}(I)$ be the set of all functions $f$ defined on $I$ satisfying the condition $|f(x)| \leq M_{f}\left(1+x^{2}\right)$, where $M_{f}$ is the constant depending only on $f$. We denote the subspace of all continuous functions belonging to $B_{x^{2}}(I)$ by $C_{x^{2}}(I)$. Let $C_{x^{2}}^{*}(I)$ be the subspace of all functions $f \in C_{x^{2}}(I)$, for which $\lim _{x \rightarrow \infty}\left(f(x) /\left(1+x^{2}\right)\right)$ is finite. The norm on $C_{x^{2}}^{*}(I)$ is

$$
\|f\|_{x^{2}}=\sup _{x \in I} \frac{|f(x)|}{1+x^{2}} .
$$

Firstly, we discuss the weighted approximation theorem.
Theorem 6. For $f \in C_{x^{2}}^{*}(I)$, we have

$$
\lim _{n \longrightarrow \infty}\left\|\widetilde{C_{n, p, q}^{\alpha, \beta, l}}(f)-f\right\|_{x^{2}}=0 .
$$

Proof. By using the Korovkin theorem, we see that it is sufficient to verify the following three conditions:

$$
\lim _{n \longrightarrow \infty}\left\|\widetilde{C_{n, p_{n}, q_{n}}^{\alpha, \beta, l}}\left(t^{i} ; x\right)-x^{i}\right\|_{x^{2}}=0, \quad i=0,1,2 .
$$

Since $\widetilde{C_{n, p_{n}, q_{n}}^{\alpha, \beta, l}}(1 ; x)=1$ and $\widetilde{C_{n, p_{n}, q_{n}}^{\alpha, \beta, l}}(t ; x)=x$, equality (34) holds true for $i=0$ and $i=1$. Finally, for $i=2$, from Lemma 2, we have

$$
\begin{gathered}
\left\|\widetilde{C_{n, p_{n}, q_{n}}^{\alpha, \beta,}}\left(t^{2} ; x\right)-x^{2}\right\| x_{x^{2}}=\sup _{x \in I} \frac{\left|\widetilde{C_{n, p_{n}, q_{n}}^{\alpha, \beta, l}}\left(t^{2} ; x\right)-x^{2}\right|}{1+x^{2}} \\
\leq \frac{p_{n}^{n+l-1} b_{n}\left(2 \alpha+[n+l]_{p_{n}, q_{n}}\right)}{\left([n]_{p_{n}, q_{n}}+\beta\right)[n+l]_{p_{n}, q_{n}}} \sup _{x \in I} \frac{x}{1+x^{2}} \\
\quad+\frac{p_{n}^{n+l-1} \alpha b_{n}^{2}\left(\alpha+[n+l]_{p_{n}, q_{n}}\right)}{\left([n]_{p_{n}, q_{n}}+\beta\right)^{2}[n+l]_{p_{n}, q_{n}}} \sup _{x \in I} \frac{1}{1+x^{2}} \\
+\frac{p_{n}^{n+l-1}}{[n+l]_{p_{n}, q_{n}}} \sup \frac{x^{2}}{1+x^{2}} \\
\leq \frac{p_{n}^{n+l-1} b_{n}\left(2 \alpha+[n+l]_{p_{n}, q_{n}}\right)}{\left([n]_{p_{n}, q_{n}}+\beta\right)[n+l]_{p_{n}, q_{n}}} \\
\quad+\frac{p_{n}^{n+l-1} \alpha b_{n}^{2}\left(\alpha+[n+l]_{p_{n}, q_{n}}\right)}{\left([n]_{p_{n}, q_{n}}+\beta\right)^{2}[n+l]_{p_{n}, q_{n}}}+\frac{p_{n}^{n+l-1}}{[n+l]_{p_{n}, q_{n}}} .
\end{gathered}
$$

We can obtain $\lim _{n \rightarrow \infty}\left\|\widetilde{C_{n, p_{n}, q_{n}}^{\alpha, \beta, l}}\left(t^{2} ; x\right)-x^{2}\right\|_{x^{2}}=0$ by using Lemma 4. Theorem 6 is proved.

We give the following definitions: The space of all real valued continuous bounded functions $f$ defined on the 
interval $I$ is denoted by $C_{B}(I)$. The norm on $C_{B}(I)$ is defined by $\|f\|=\sup \{|f(x)|: x \in I\}$. The Peetre's $K$-functional is given by

$$
K_{2}(f ; \delta)=\inf _{g \in W^{2}}\left\{\|f-g\|+\delta\left\|g^{\prime \prime}\right\|\right\},
$$

where $\delta>0$ and $W^{2}=\left\{g \in C_{B}[0, \infty): g^{\prime}, g^{\prime \prime} \in C_{B}[0, \infty)\right\}$. For $f \in C_{B}[0, \infty)$, the usual modulus of continuity and the second order modulus of smoothness are defined as follows:

$$
\begin{aligned}
& \omega(f ; \delta)=\sup _{0<h \leq \delta} \sup _{x \in[0, \infty)}|f(x+h)-f(x)|, \\
& \begin{array}{c}
\omega_{2}(f ; \sqrt{\delta}) \\
\quad=\sup _{0<h \leq \delta} \sup _{x \in[0, \infty)}|f(x+2 h)-2 f(x+h)+f(x)| .
\end{array}
\end{aligned}
$$

By [33], there exists a constant $C>0$, such that

$$
K_{2}(f ; \delta) \leq C \omega_{2}(f ; \sqrt{\delta}) .
$$

Now, we establish local approximation theorems as follows.

Theorem 7. For $f \in C_{B}(I)$, we have

$$
\begin{aligned}
& \left|\widetilde{C_{n, p, q}^{\alpha, \beta, l}}(f ; x)-f(x)\right| \\
& \quad \leq C \omega_{2}\left(f ; \sqrt{\frac{p^{n+l-1} b_{n}\left(2 \alpha+[n+l]_{p, q}\right) x}{2\left([n]_{p, q}+\beta\right)[n+l]_{p, q}}}\right),
\end{aligned}
$$

where $C$ is a positive constant.

Proof. Let $g \in W^{2}$; by Taylor's expansion, we have

$$
g(t)=g(x)+g^{\prime}(x)(t-x)+\int_{x}^{t}(t-u) g^{\prime \prime}(u) d u,
$$

and, applying $\widetilde{C_{n, p, q}^{\alpha, \beta, l}}$ to (41), using (9) and (12), we get

$$
\begin{aligned}
& \widetilde{C_{n, p, q}^{\alpha, \beta, l}}(g ; x)-g(x) \\
& =\widetilde{C_{n, p, q}^{\alpha, \beta, l}}\left(\int_{x}^{t}(t-u) g^{\prime \prime}(u) d u ; x\right) .
\end{aligned}
$$

Thus, from (13), we have

$$
\begin{aligned}
& \left|\widetilde{C_{n, p, q}^{\alpha, \beta, l}}(g ; x)-g(x)\right| \\
& =\left|\widetilde{C_{n, p, q}^{\alpha, \beta, l}}\left(\int_{x}^{t}(t-u) g^{\prime \prime}(u) d u ; x\right)\right| \\
& \leq \widetilde{C_{n, p, q}^{\alpha, \beta, l}}\left(\left|\int_{x}^{t}\right| t-u|| g^{\prime \prime}(u)|d u| ; x\right) \\
& \leq \widetilde{C_{n, p, q}^{\alpha, \beta, l}}\left((t-x)^{2} ; x\right)\left\|g^{\prime \prime}\right\| \\
& \leq \frac{p^{n+l-1} b_{n}\left(2 \alpha+[n+l]_{p, q}\right) x}{\left([n]_{p, q}+\beta\right)[n+l]_{p, q}}\left\|g^{\prime \prime}\right\| .
\end{aligned}
$$

On the other hand, by (3) and (9), we have

$$
\begin{aligned}
& \widetilde{C_{n, p, q}^{\alpha, \beta, l}}(f ; x) \\
& \quad \leq \sum_{k=0}^{n+l} c_{n, p, q}^{k, l}(u(x))\left|f\left(\frac{p^{n+l-k}[k]_{p, q}+\alpha}{[n]_{p, q}+\beta} b_{n}\right)\right| \\
& \leq\|f\| .
\end{aligned}
$$

Now (43) and (44) imply

$$
\begin{aligned}
& \left|\widetilde{C_{n, p, q}^{\alpha, \beta, l}}(f ; x)-f(x)\right| \\
& \leq\left|\widetilde{C_{n, p, q}^{\alpha, \beta, l}}(f-g ; x)-(f-g)(x)\right| \\
& \quad+\left|\widetilde{C_{n, p, q}^{\alpha, \beta, l}}(g ; x)-g(x)\right| \\
& \leq 2\|f-g\|+\frac{p^{n+l-1} b_{n}\left(2 \alpha+[n+l]_{p, q}\right) x}{\left([n]_{p, q}+\beta\right)[n+l]_{p, q}}\left\|g^{\prime \prime}\right\|,
\end{aligned}
$$

and, from (36), taking infimum on the right hand side over all $g \in W^{2}$, we obtain

$$
\begin{aligned}
& \left|\widetilde{C_{n, p, q}^{\alpha, \beta, l}}(f ; x)-f(x)\right| \\
& \quad \leq 2 K_{2}\left(f ; \frac{p^{n+l-1} b_{n}\left(2 \alpha+[n+l]_{p, q}\right) x}{2\left([n]_{p, q}+\beta\right)[n+l]_{p, q}}\right) .
\end{aligned}
$$

Finally, using (39), we get

$$
\begin{aligned}
& \left|\widetilde{C_{n, p, q}^{\alpha, \beta, l}}(f ; x)-f(x)\right| \\
& \quad \leq C \omega_{2}\left(f ; \sqrt{\frac{p^{n+l-1} b_{n}\left(2 \alpha+[n+l]_{p, q}\right) x}{2\left([n]_{p, q}+\beta\right)[n+l]_{p, q}}}\right) .
\end{aligned}
$$

Theorem 7 is proved.

Theorem 8. For $f \in C_{B}(I)$ and $\delta>0$, we have

$$
\begin{aligned}
& \left|\widetilde{C_{n, p, q}^{\alpha, \beta, l}}(f ; x)-f(x)\right| \\
& \quad \leq\left(1+\frac{1}{\delta} \sqrt{\frac{p^{n+l-1} b_{n}\left(2 \alpha+[n+l]_{p, q}\right) x}{\left([n]_{p, q}+\beta\right)[n+l]_{p, q}}}\right) \omega(f ; \delta) .
\end{aligned}
$$

Proof. Since

$$
\begin{aligned}
|f(t)-f(x)| & \leq \omega(f ;|t-x|) \\
& \leq\left(1+\frac{|t-x|}{\delta}\right) \omega(f ; \delta),
\end{aligned}
$$

applying $\widetilde{C_{n, p, q}^{\alpha, \beta, l}}(f ; x)$ to (49), we obtain

$$
\begin{aligned}
& \left|\widetilde{C_{n, p, q}^{\alpha, \beta, l}}(f ; x)-f(x)\right| \leq \widetilde{C_{n, p, q}^{\alpha, \beta, l}}(|f(t)-f(x)| ; x) \\
& \quad \leq\left(1+\frac{1}{\delta} \widetilde{C_{n, p, q}^{\alpha, \beta, l}}(|t-x| ; x)\right) \omega(f ; \delta),
\end{aligned}
$$


using Cauchy-Schwartz inequality, we have

$$
\begin{aligned}
& \left|\widetilde{C_{n, p, q}^{\alpha, \beta, l}}(f ; x)-f(x)\right| \\
& \quad \leq\left[1+\frac{1}{\delta} \sqrt{\left.\widetilde{C_{n, p, q}^{\alpha, \beta, l}}\left((t-x)^{2} ; x\right)\right] \omega(f ; \delta)}\right. \\
& \quad \leq\left(1+\frac{1}{\delta} \sqrt{\frac{p^{n+l-1} b_{n}\left(2 \alpha+[n+l]_{p, q}\right) x}{\left([n]_{p, q}+\beta\right)[n+l]_{p, q}}}\right) \omega(f ; \delta) .
\end{aligned}
$$

Theorem 8 is proved.

Corollary 9. From Theorem 8 , applied to $\delta=$ $\sqrt{p^{n+l-1} b_{n}\left(2 \alpha+[n+l]_{p, q}\right) x /\left([n]_{p, q}+\beta\right)[n+l]_{p, q}}$, we have

$$
\begin{aligned}
& \left|\widetilde{C_{n, p, q}^{\alpha, \beta, l}}(f ; x)-f(x)\right| \\
& \quad \leq 2 \omega\left(f ; \sqrt{\frac{p^{n+l-1} b_{n}\left(2 \alpha+[n+l]_{p, q}\right) x}{\left([n]_{p, q}+\beta\right)[n+l]_{p, q}}}\right) .
\end{aligned}
$$

Remark 10. For any fixed $x \in I$, we have $\lim _{n \rightarrow \infty}\left(p_{n}^{n+l-1} b_{n}(2 \alpha\right.$ $\left.\left.+[n+l]_{p_{n}, q_{n}}\right) x /\left([n]_{p_{n}, q_{n}}+\beta\right)[n+l]_{p_{n}, q_{n}}\right)=0$, and this gives us a rate of pointwise convergence of the operators $\widetilde{C_{n, p, q}^{\alpha, \beta, l}}(f ; x)$ to $f(x)$.

Theorem 11. If $f$ is differentiable on $I$ and $f^{\prime} \in C_{B}(I)$, then for $\delta>0$, we have

$$
\begin{aligned}
& \left|\widetilde{C_{n, p, q}^{\alpha, \beta, l}}(f ; x)-f(x)\right| \\
& \quad \leq \sqrt{\frac{p^{n+l-1} b_{n}\left(2 \alpha+[n+l]_{p, q}\right) x}{\left([n]_{p, q}+\beta\right)[n+l]_{p, q}}}(1 \\
& \left.+\frac{1}{\delta} \sqrt{\frac{p^{n+l-1} b_{n}\left(2 \alpha+[n+l]_{p, q}\right) x}{\left([n]_{p, q}+\beta\right)[n+l]_{p, q}}}\right) \omega\left(f^{\prime} ; \delta\right) .
\end{aligned}
$$

Proof. Since $f(t)=f(x)+f^{\prime}(x)(t-x)+f(t)-f(x)-f^{\prime}(x)(t-$ $x)$ and we have (12), we can write

$$
\begin{aligned}
& \left|\widetilde{C_{n, p, q}^{\alpha, \beta, l}}(f ; x)-f(x)\right| \\
& \quad \leq \widetilde{C_{n, p, q}^{\alpha, \beta, l}}\left(\left|f(t)-f(x)-f^{\prime}(x)(t-x)\right| ; x\right),
\end{aligned}
$$

and, by the mean value theorem, we obtain

$$
\begin{aligned}
& \left|f(t)-f(x)-f^{\prime}(x)(t-x)\right| \\
& \quad \leq|t-x|\left(1+\frac{|t-x|}{\delta}\right) \omega\left(f^{\prime} ; \delta\right),
\end{aligned}
$$

so, by the Cauchy-Schwartz inequality, we get

$$
\begin{aligned}
& \widetilde{C_{n, p, q}^{\alpha, \beta, l}}\left(\left|f(t)-f(x)-f^{\prime}(x)(t-x)\right| ; x\right) \\
& \quad \leq\left[\widetilde{C_{n, p, q}^{\alpha, \beta, l}}(|t-x| ; x)+\frac{1}{\delta} \widetilde{C_{n, p, q}^{\alpha, \beta, l}}\left((t-x)^{2} ; x\right)\right] \\
& \cdot \omega\left(f^{\prime} ; \delta\right) \leq\left[\sqrt{\widetilde{C_{n, p, q}^{\alpha, \beta, l}}\left((t-x)^{2} ; x\right)}\right. \\
& \left.+\frac{1}{\delta} \widetilde{C_{n, p, q}^{\alpha, \beta, l}}\left((t-x)^{2} ; x\right)\right] \omega\left(f^{\prime} ; \delta\right) \\
& \quad \leq \sqrt{\widetilde{C_{n, p, q}^{\alpha, \beta, l}}\left((t-x)^{2} ; x\right)}[1 \\
& \left.+\frac{1}{\delta} \sqrt{\widetilde{C_{n, p, q}^{\alpha, \beta, l}}\left((t-x)^{2} ; x\right)}\right] \omega\left(f^{\prime} ; \delta\right),
\end{aligned}
$$

and then we have the desired result by (13).

Corollary 12. From Theorem 11, let $f \in C_{B}(I)$, applied to $\delta=\sqrt{p^{n+l-1} b_{n}\left(2 \alpha+[n+l]_{p, q}\right) x /\left([n]_{p, q}+\beta\right)[n+l]_{p, q}}$; then we have

$$
\begin{aligned}
& \left|\widetilde{C_{n, p, q}^{\alpha, \beta, l}}(f ; x)-f(x)\right| \\
& \quad \leq 2 \sqrt{\frac{p^{n+l-1} b_{n}\left(2 \alpha+[n+l]_{p, q}\right) x}{\left([n]_{p, q}+\beta\right)[n+l]_{p, q}}} \omega\left(f^{\prime} ;\right. \\
& \left.\sqrt{\frac{p^{n+l-1} b_{n}\left(2 \alpha+[n+l]_{p, q}\right) x}{\left([n]_{p, q}+\beta\right)[n+l]_{p, q}}}\right) .
\end{aligned}
$$

Next, we study the rate of convergence of the operators $\widetilde{C_{n, p, q}^{\alpha, \beta, l}}(f ; x)$ with the help of functions of Lipschitz class $\operatorname{Lip}_{M}(\mu)$, where $M>0$ and $0<\mu \leq 1$. A function $f$ belongs to $\operatorname{Lip}_{M}(\mu)$ if

$$
|f(y)-f(x)| \leq M|y-x|^{\mu} \quad(x, y \in \mathbb{R}) .
$$

We have the following theorem.

Theorem 13. Let $f \in \operatorname{Lip}_{M}(\mu), 0<\mu \leq 1$; we have

$$
\begin{aligned}
& \left|\widetilde{C_{n, p, q}^{\alpha, \beta, l}}(f ; x)-f(x)\right| \\
& \quad \leq M\left[\frac{p^{n+l-1} b_{n}\left(2 \alpha+[n+l]_{p, q}\right) x}{\left([n]_{p, q}+\beta\right)[n+l]_{p, q}}\right]^{\mu / 2} .
\end{aligned}
$$


Proof. Obviously, $\widetilde{C_{n, p, q}^{\alpha, \beta, l}}(f ; x)$ are linear positive operators; since $f \in \operatorname{Lip}_{M}(\mu)(0<\mu \leq 1)$, we have

$$
\begin{aligned}
& \left|\widetilde{C_{n, p, q}^{\alpha, \beta, l}}(f ; x)-f(x)\right| \leq \widetilde{C_{n, p, q}^{\alpha, \beta, l}}(|f(t)-f(x)| ; x) \\
& =\sum_{k=0}^{n+l} c_{n, p, q}^{k, l}(u(x)) \mid f\left(\frac{p^{n+l-k}[k]_{p, q}+\alpha}{[n]_{p, q}+\beta} b_{n}\right) \\
& -f(x)\left|\leq M \sum_{k=0}^{n+l} c_{n, p, q}^{k, l}(u(x))\right| \frac{p^{n+l-k}[k]_{p, q}+\alpha}{[n]_{p, q}+\beta} b_{n} \\
& -\left.x\right|^{\mu} \leq M \sum_{k=0}^{n+l}\left[c_{n, p, q}^{k, l}(u(x))\right. \\
& \left.\cdot\left(\frac{p^{n+l-k}[k]_{p, q}+\alpha}{[n]_{p, q}+\beta} b_{n}-x\right)^{2}\right]^{\mu / 2} \\
& \cdot\left[c_{n, p, q}^{k, l}(u(x))\right]^{(2-\mu) / 2} .
\end{aligned}
$$

Applying Hölder's inequality for sums, we obtain

$$
\begin{aligned}
& \left|\widetilde{C_{n, p, q}^{\alpha, \beta, l}}(f ; x)-f(x)\right| \leq M\left[\sum_{k=0}^{n+l} c_{n, p, q}^{k, l}(u(x))\right. \\
& \left.\cdot\left(\frac{p^{n+l-k}[k]_{p, q}+\alpha}{[n]_{p, q}+\beta} b_{n}-x\right)^{2}\right]^{\mu / 2}\left[\sum_{k=0}^{n+l} c_{n, p, q}^{k, l}(u(x))\right]^{(2-\mu) / 2} \\
& =M\left[\widetilde{C_{n, p, q}^{\alpha, \beta, l}}\left((t-x)^{2} ; x\right)\right]^{\mu / 2} \\
& \leq M\left[\frac{p^{n+l-1} b_{n}\left(2 \alpha+[n+l]_{p, q}\right) x}{\left([n]_{p, q}+\beta\right)[n+l]_{p, q}}\right]^{\mu / 2}
\end{aligned}
$$

Theorem 13 is proved.

Now, we give a Voronovskaja-type asymptotic formula for $\widetilde{C_{n, p, q}^{\alpha, \beta, l}}(f ; x)$.

Theorem 14. For $x \in I$, we have the following conclusion:

$$
\lim _{n \longrightarrow \infty} \frac{[n]_{p_{n}, q_{n}}}{b_{n}}\left(\widetilde{C_{n, p_{n}}^{\alpha, \beta, q_{n}}}(f ; x)-f(x)\right)=\frac{f^{\prime \prime}(x)}{2} a x .
$$

Proof. Let $x \in I$ be fixed. By the Taylor formula, we may write

$$
\begin{aligned}
f(t)= & f(x)+f^{\prime}(x)(t-x)+\frac{1}{2} f^{\prime \prime}(x)(t-x)^{2} \\
& +r(t ; x)(t-x)^{2},
\end{aligned}
$$

where $r(t ; x)$ is the Peano form of the remainder, $r(t ; x) \epsilon$ $C(I)$; using L'Hospital's rule, we have

$$
\begin{aligned}
& \lim _{t \rightarrow x} r(t ; x) \\
& =\lim _{t \rightarrow x} \frac{f(t)-f(x)-f^{\prime}(x)(t-x)-(1 / 2) f^{\prime \prime}(x)(t-x)^{2}}{(t-x)^{2}} \\
& =\lim _{t \rightarrow x} \frac{f^{\prime}(t)-f^{\prime}(x)-f^{\prime \prime}(x)(t-x)}{2(t-x)} \\
& =\lim _{t \rightarrow x} \frac{f^{\prime \prime}(t)-f^{\prime \prime}(x)}{2}=0 .
\end{aligned}
$$

Since we have (12), applying $\widetilde{C_{n, p, q}^{\alpha, \beta, l}}(f ; x)$ to (63), we obtain

$$
\begin{aligned}
& \frac{[n]_{p, q}}{b_{n}}\left(\widetilde{C_{n, p, q}^{\alpha, \beta, l}}(f ; x)-f(x)\right) \\
& =\frac{f^{\prime \prime}(x)}{2} \frac{[n]_{p, q}}{b_{n}} \widetilde{C_{n, p, q}^{\alpha, \beta, l}}\left((t-x)^{2} ; x\right) \\
& \quad+\frac{[n]_{p, q}}{b_{n}} \widetilde{C_{n, p, q}^{\alpha, \beta, l}}\left(r(t ; x)(t-x)^{2} ; x\right) .
\end{aligned}
$$

By the Cauchy-Schwarz inequality, we have

$$
\begin{aligned}
& \widetilde{C_{n, p, q}^{\alpha, \beta, l}}\left(r(t ; x)(t-x)^{2} ; x\right) \\
& \quad \leq \sqrt{\widetilde{{C_{n, p, q}^{\alpha, \beta, l}}_{1}}\left(r^{2}(t ; x) ; x\right)} \sqrt{\overline{{C_{n, p, q}^{\alpha, \beta, l}}_{1}}\left((t-x)^{4} ; x\right)} .
\end{aligned}
$$

From $r^{2}(x ; x)=0$ and $(17)$, we get $\lim _{n \rightarrow \infty}\left([n]_{p_{n}, q_{n}}\right.$ ' $\left.b_{n}\right) \widetilde{C_{n, p_{n}, q_{n}}^{\alpha, \beta, l}}\left(r(t ; x)(t-x)^{2} ; x\right)=0$. Hence, from (16), we have

$$
\begin{aligned}
& \lim _{n \rightarrow \infty} \frac{[n]_{p_{n}, q_{n}}}{b_{n}}\left(\widetilde{C_{n, p_{n}, q_{n}}^{\alpha, \beta, l}}(f ; x)-f(x)\right) \\
& \quad=\lim _{n \longrightarrow \infty} \frac{f^{\prime \prime}(x)}{2} \frac{[n]_{p_{n}, q_{n}}}{b_{n}} \widetilde{C_{n, p_{n}, q_{n}}^{\alpha, \beta, l}}\left((t-x)^{2} ; x\right) \\
& \quad=\frac{f^{\prime \prime}(x)}{2} a x .
\end{aligned}
$$

Theorem 14 is proved.

Remark 15. For the function $f(t)=t^{2}$, from Theorem 14, we have the limit equality

$$
\begin{aligned}
& \lim _{n \longrightarrow \infty} \frac{[n]_{p_{n}, q_{n}}}{b_{n}}\left(\widetilde{C_{n, p_{n}, q_{n}}^{\alpha, \beta, l}}\left(t^{2} ; x\right)-x^{2}\right)=\frac{\left(x^{2}\right)^{\prime \prime}}{2} a x \\
& \quad=a x,
\end{aligned}
$$

which is the corresponding result in (16).

\section{Graphical and Numerical Analysis I}

In this section, we give several graphs and numerical examples to show the convergence of $\widetilde{C_{n, p, q}^{\alpha, \beta, l}}(f ; x)$ to $f(x)$ with different values of parameters which satisfy the conclusions of Lemmas 4 and 5. 
TABLE 1: The absolute error bound of the approximation of $\widetilde{C_{n, p, q}^{\alpha, \beta, l}}(f ; x)$ with $\alpha=1, \beta=2, l=5, b_{n}=10, p=0.999999$ and different values of $n$ and $q$.

\begin{tabular}{|c|c|c|c|c|}
\hline \multirow[t]{2}{*}{$q$} & \multicolumn{3}{|c|}{$f(x)-\widetilde{C_{n, p, q}^{\alpha, \beta, l}}(f ; x) \|$} & \multirow[b]{2}{*}{$n=100$} \\
\hline & $n=30$ & $n=50$ & $n=80$ & \\
\hline 0.99 & 0.215524 & 0.169329 & 0.132302 & 0.118517 \\
\hline 0.999 & 0.202854 & 0.146061 & 0.101294 & 0.084213 \\
\hline 0.9999 & 0.201487 & 0.143719 & 0.098331 & 0.081025 \\
\hline 0.99999 & 0.201349 & 0.143485 & 0.098037 & 0.080710 \\
\hline
\end{tabular}

TABLE 2: The absolute error bound of the approximation of $\widetilde{C_{n, p, q}^{\alpha, \beta, l}}(f ; x)$ with $\alpha=1, \beta=2, l=5, b_{n}=10$ and different values of $n$, $p$, and $q$, where $p=1-1 / 10^{m}, q=1-1 / 10^{m-1}$.

\begin{tabular}{|c|c|c|c|c|}
\hline \multirow{2}{*}{$m$} & \multicolumn{4}{|c|}{$\left\|f(x)-\widetilde{C_{n, p, q}^{\alpha, \beta, l}}(f ; x)\right\|_{\infty}$} \\
\hline & $n=30$ & $n=50$ & $n=80$ & $n=100$ \\
\hline 6 & 0.20134927 & 0.14348509 & 0.09803662 & 0.08070974 \\
\hline 7 & 0.20134028 & 0.14346631 & 0.09801166 & 0.08068261 \\
\hline 8 & 0.20133938 & 0.14346444 & 0.09800917 & 0.08067989 \\
\hline 9 & 0.20133930 & 0.14346425 & 0.09800892 & 0.08067962 \\
\hline 10 & 0.20133929 & 0.14346423 & 0.09800889 & 0.08067960 \\
\hline
\end{tabular}

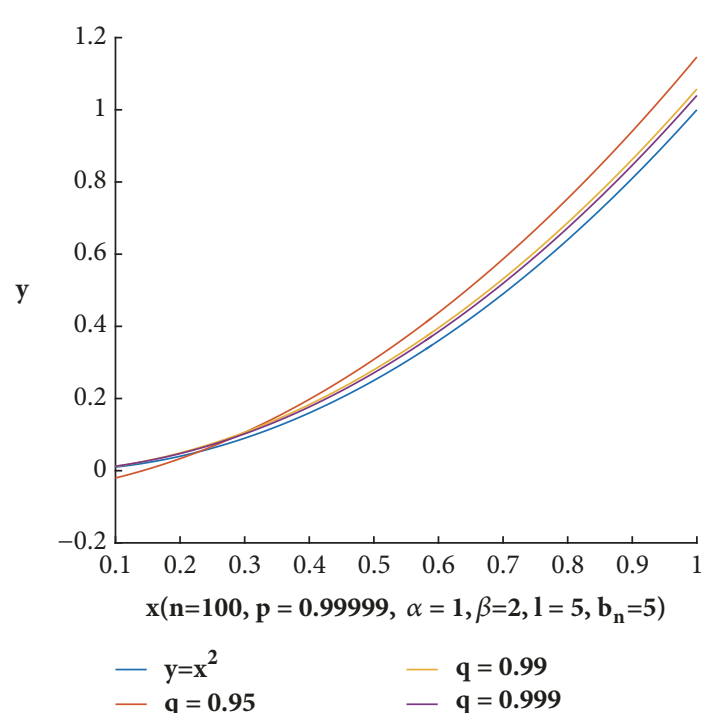

FIGURE 1: The figures of $\widetilde{C_{n, p, q}^{\alpha, \beta, l}}(f ; x)$ with $n=100, p=0.99999, \alpha=1$, $\beta=2, l=5, b_{n}=5$ and different values of $q$.

Example 16. Let $f(x)=x^{2}$; the graphs of $\widetilde{C_{n, p, q}^{\alpha, \beta, l}}(f ; x)$ with $n=100, p=0.99999, \alpha=1, \beta=2, l=5, b_{n}=5$ and different values of $q$ are shown in Figure 1. The graphs of $\widetilde{C_{n, p, q}^{\alpha, \beta, l}}(f ; x)$ with $p=0.99999, q=0.99, \alpha=1, \beta=2, l=5$, $b_{n}=10$ and different values of $n$ are shown in Figure 2. The graphs of $\widetilde{C_{n, p, q}^{\alpha, \beta, l}}(f ; x)$ with $n=100, \alpha=1, \beta=2, l=5$, $b_{n}=10$ and different values of $p$ and $q$ are shown in Figure 3 . Moreover, we give a comparison on the approximation of $\widetilde{C_{n, p, q}^{\alpha, \beta, l}}(f ; x)$ (the red one) and $C_{n, m}^{(\alpha, \beta)}(f ; x, p, q)$ (the yellow one) in Figure 4. In Tables 1 and 2, we show the absolute error bound of the approximation of $\widetilde{C_{n, p, q}^{\alpha, \beta, l}}(f ; x)$ with $\alpha=1, \beta=2$, $l=5, b_{n}=10$ and different values of $n, p$, and $q$ to $f(x)$.

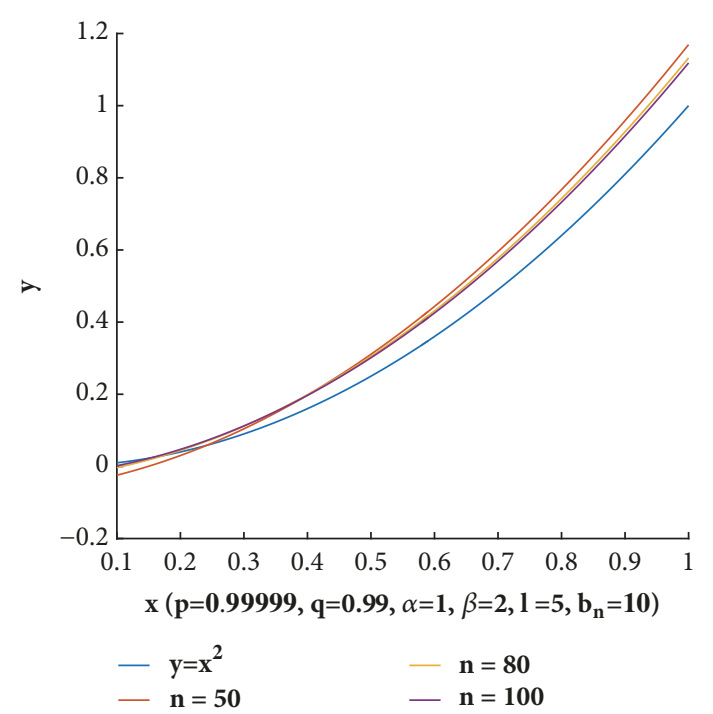

Figure 2: The figures of $\widetilde{C_{n, p, q}^{\alpha, \beta, l}}(f ; x)$ with $p=0.99999, q=0.99$, $\alpha=1, \beta=2, l=5, b_{n}=10$ and different values of $n$.

\section{Construction of Bivariate Operators and Weighted Approximation Properties}

We introduce the bivariate tensor product $(p, q)$-analogue of Chlodowsky type Bernstein-Stancu-Schurer operators as follows:

$$
\begin{aligned}
& C_{p_{n_{1}}, p_{n_{2}}, q_{n_{1}}, q_{n_{2}}}^{n_{1}, n_{2}, \alpha ; \beta,}(f ; x) \\
& \quad=\sum_{k_{1}=0}^{n_{1}+l} \sum_{k_{2}=0}^{n_{2}+l} c_{n_{1}, p_{n_{1}}, q_{n_{1}}}^{k, l}(u(x)) c_{n_{2}, p_{n_{2}}, q_{n_{2}}}^{k, l}(v(y))
\end{aligned}
$$




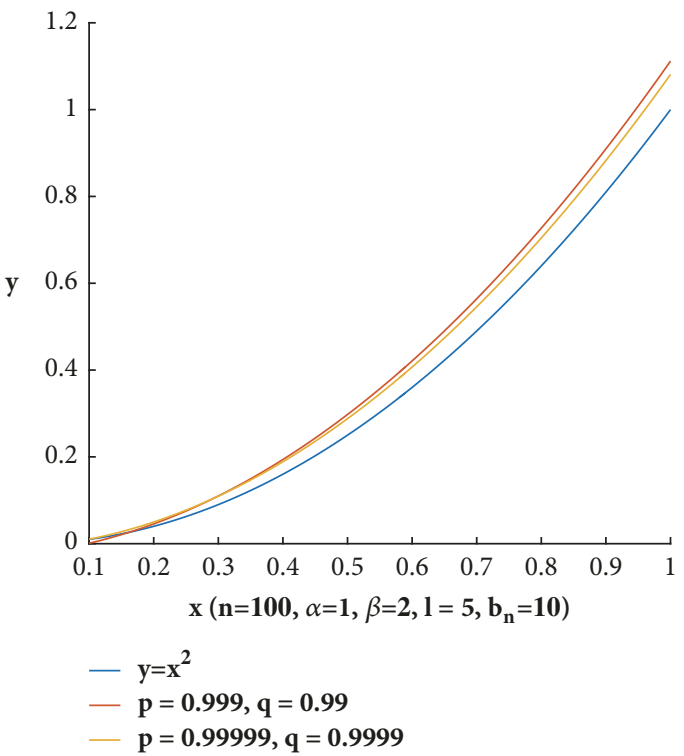

Figure 3: The figures of $\widetilde{C_{n, p, q}^{\alpha, \beta, l}}(f ; x)$ with $n=100, \alpha=1, \beta=2, l=$ $5, b_{n}=10$ and different values of $p$ and $q$.

$$
\begin{aligned}
& \times f\left(\frac{p_{n_{1}}^{n_{1}+l-k_{1}}\left[k_{1}\right]_{p_{n_{1}}, q_{n_{1}}}+\alpha}{\left[n_{1}\right]_{p_{n_{1}}, q_{n_{1}}}+\beta}\right. \\
& \left.\cdot b_{n_{1}}, \frac{p_{n_{2}}^{n_{2}+l-k_{2}}\left[k_{2}\right]_{p_{n_{2}}, q_{n_{2}}}+\alpha}{\left[n_{2}\right]_{p_{n_{2}}, q_{n_{2}}}+\beta} b_{n_{2}}\right),
\end{aligned}
$$

where

$$
\begin{aligned}
& u(x)= \frac{\left(\left[n_{1}\right]_{p_{n_{1}}, q_{n_{1}}}+\beta\right) x-\alpha b_{n_{1}}}{\left[n_{1}+l\right]_{p_{n_{1}}, q_{n_{1}}}}, \\
&\left(\frac{\alpha b_{n_{1}}}{\left[n_{1}\right]_{p_{n_{1}}, q_{n_{1}}}+\beta} \leq x \leq \frac{\left[n_{1}+l\right]_{p_{n_{1}}, q_{n_{1}}}+\alpha}{\left[n_{1}\right]_{p_{n_{1}}, q_{n_{1}}}+\beta} b_{n_{1}}\right), \\
& v(y)= \frac{\left(\left[n_{2}\right]_{p_{n_{2}}, q_{n_{2}}}+\beta\right) y-\alpha b_{n_{2}}}{\left[n_{2}+l\right]_{p_{n_{2}}, q_{n_{2}}}}, \\
&\left(\frac{\alpha b_{n_{2}}}{\left[n_{2}\right]_{p_{n_{2}}, q_{n_{2}}}+\beta} \leq y \leq \frac{\left[n_{2}+l\right]_{p_{n_{2}}, q_{n_{2}}}+\alpha}{\left[n_{2}\right]_{p_{n_{2}}, q_{n_{2}}}+\beta} b_{n_{2}}\right), \\
& c_{n, p, q}^{k, l}(x)=\frac{1}{p^{(n+l)(n+l-1) / 2}}\left[\begin{array}{c}
k+l]_{p, q} \\
p^{k(k-1) / 2}\left(\frac{x}{b_{n}}\right)^{k} \\
\cdot\left(1-\frac{x}{b_{n}}\right)_{p, q}^{n+l-k},
\end{array}\right.
\end{aligned}
$$

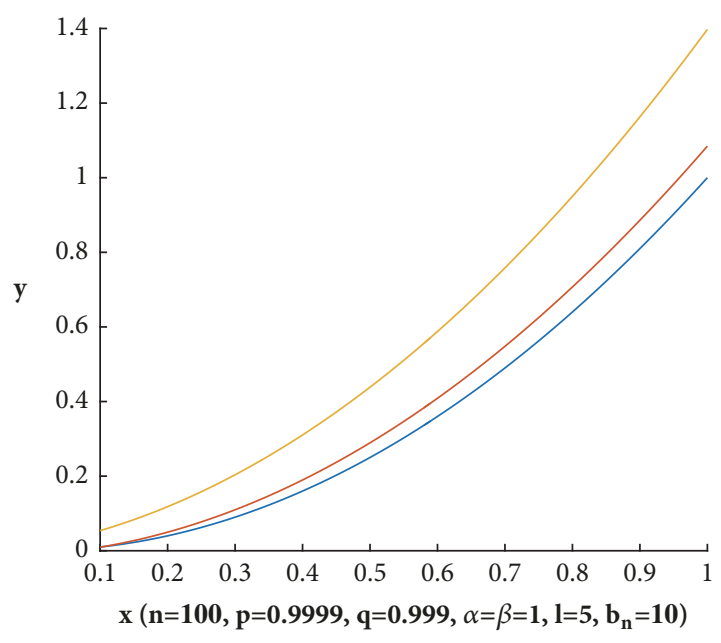

Figure 4: The figures of $\widetilde{C_{n, p, q}^{\alpha, \beta, l}}(f ; x)$ (the red one) and $C_{n, m}^{(\alpha, \beta)}(f ; x$, $p, q)$ (the yellow one) with $n=100, b_{n}=10, \alpha=\beta=1, m=l=$ $5, p=0.9999, q=0.999$.

$0<q_{n_{1}}, q_{n_{2}}<p_{n_{1}}, p_{n_{2}} \leq 1,0 \leq \alpha \leq \beta$, and $\left\{b_{n_{1}}\right\},\left\{b_{n_{2}}\right\}$ are increasing sequences of positive terms with $b_{n_{1}}, b_{n_{2}} \longrightarrow \infty$, $b_{n_{1}} /\left[n_{1}\right]_{p_{n_{1}}, q_{n_{1}}}, b_{n_{2}} /\left[n_{2}\right]_{p_{n_{2}}, q_{n_{2}}} \longrightarrow 0$ as $n_{1}, n_{2} \longrightarrow \infty$.

Lemma 17. Let $e_{i, j}(x, y)=x^{i} y^{j}, i, j \in \mathbb{N},(x, y) \in I \times I$ be the two-dimensional test functions; using Lemma 1, we easily obtain the following equalities:

$$
\begin{aligned}
& C_{p_{n_{1}}, p_{n_{2}}, q_{n_{1}}, q_{n_{2}}}^{n_{1}, \widetilde{n_{2}, \alpha, \beta, l}}\left(e_{0,0} ; x, y\right)=1, \\
& C_{p_{n_{1}}, p_{n_{2}}, q_{n_{1}}, q_{n_{2}}}^{n_{1}, n_{2}, \alpha, \beta, l}
\end{aligned}
$$

$$
C_{p_{n_{1}}, p_{n_{2}}, q_{n_{1}}, q_{n_{2}}}^{n_{1}, n_{2}, \alpha, \beta, l} \quad\left(e_{0,1} ; x, y\right)=y
$$

$C_{p_{n_{1}}, p_{n_{2}}, q_{n_{1}}, q_{n_{2}}}^{n_{1}, n_{2}, \alpha, \beta, l}\left(e_{1,1} ; x, y\right)=x y$

$C_{p_{n_{1}}, p_{n_{2}}, q_{n_{1}}, q_{n_{2}}}^{n_{1}, n_{2}, \alpha, \beta, l}\left(e_{2,0} ; x, y\right)$

$$
\begin{aligned}
= & x^{2}-\frac{p_{n_{1}}^{n_{1}+l-1} x^{2}}{\left[n_{1}+l\right]_{p_{n_{1}}, q_{n_{1}}}} \\
& +\frac{p_{n_{1}}^{n_{1}+l-1} b_{n_{1}}\left(2 \alpha+\left[n_{1}+l\right]_{p_{n_{1}}, q_{n_{1}}}\right) x}{\left(\left[n_{1}\right]_{p_{n_{1}}, q_{n_{1}}}+\beta\right)\left[n_{1}+l\right]_{p_{n_{1}}, q_{n_{1}}}} \\
& -\frac{p_{n_{1}}^{n_{1}+l-1} \alpha b_{n_{1}}^{2}\left(\alpha+\left[n_{1}+l\right]_{p_{n_{1}}, q_{n_{1}}}\right)}{\left(\left[n_{1}\right]_{p_{n_{1}}, q_{n_{1}}}+\beta\right)^{2}\left[n_{1}+l\right]_{p_{n_{1}}, q_{n_{1}}}}
\end{aligned}
$$

$$
\begin{gathered}
C_{p_{n_{1}}, p_{n_{2}}, q_{n_{1}}, q_{n_{2}}}^{n_{1}, n_{2}, \alpha, \beta, l}\left(e_{0,2} ; x, y\right) \\
=y^{2}-\frac{p_{n_{2}}^{n_{2}+l-1} y^{2}}{\left[n_{2}+l\right]_{p_{n_{2}}, q_{n_{2}}}}
\end{gathered}
$$




$$
\begin{array}{r}
+\frac{p_{n_{2}}^{n_{2}+l-1} b_{n_{2}}\left(2 \alpha+\left[n_{2}+l\right]_{p_{n_{2}}, q_{n_{2}}}\right) y}{\left(\left[n_{2}\right]_{p_{n_{2}}, q_{n_{2}}}+\beta\right)\left[n_{2}+l\right]_{p_{n_{2}}, q_{n_{2}}}} \\
-\frac{p_{n_{2}}^{n_{2}+l-1} \alpha b_{n_{2}}^{2}\left(\alpha+\left[n_{2}+l\right]_{p_{n_{2}}, q_{n_{2}}}\right)}{\left(\left[n_{2}\right]_{p_{n_{2}}, q_{n_{2}}}+\beta\right)^{2}\left[n_{2}+l\right]_{p_{n_{2}}, q_{n_{2}}}} .
\end{array}
$$

Lemma 18. Using Lemma 17 and (16), (17) of Lemma 5, we have the following statements:

$$
\begin{aligned}
& C_{p_{n_{1}}, p_{n_{2}}, q_{n_{1}}, q_{n_{2}}}^{n_{1}, n_{2}, \alpha, \beta} \quad(t-x ; x, y)=0, \\
& C_{p_{n_{1}}, p_{n_{2}}, q_{n_{1}}, q_{n_{2}}}^{n_{1}, n_{2}, \alpha, l} \quad(s-y ; x, y)=0, \\
& C_{p_{n_{1}}, p_{n_{2}}, q_{n_{1}}, q_{n_{2}}}^{n_{1}, n_{2}, \alpha, l}\left((t-x)^{2} ; x, y\right)=O\left(\frac{b_{n_{1}}}{\left[n_{1}\right]_{p_{n_{1}}, q_{n_{1}}}}\right) x \\
& C_{p_{n_{1}}, p_{n_{2}}, q_{n_{1}}, q_{n_{2}}}^{n_{1}, n_{2}, \beta, l}\left((s-y)^{2} ; x, y\right)=O\left(\frac{b_{n_{2}}}{\left[n_{2}\right]_{p_{n_{2}}, q_{n_{2}}}}\right) y, \\
& C_{p_{n_{1}}, p_{n_{2}}, q_{n_{1}}, q_{n_{2}}}^{n_{1}, \widetilde{n_{2}, \alpha, \beta, l}}\left((t-x)^{4} ; x, y\right)=O\left(\frac{b_{n_{1}}^{2}}{\left[n_{1}\right]_{p_{n_{1}}, q_{n_{1}}}^{2}}\right) x^{2}, \\
& C_{p_{n_{1}}, p_{n_{2}}, q_{n_{1}}, q_{n_{2}}}^{n_{1}, \widetilde{n_{2}, \alpha, \beta, l}}\left((s-y)^{4} ; x, y\right)=O\left(\frac{b_{n_{2}}^{2}}{\left[n_{2}\right]_{p_{n_{2}}, q_{n_{2}}}^{2}}\right) y^{2} .
\end{aligned}
$$

Let $B_{\rho}$ be the space of all functions $f$ defined on $I \times I$ satisfying the condition $|f(x)| \leq M_{f} \rho(x, y)$, where $M_{f}$ is a positive constant depending only on $f$ and $\rho(x, y)=$ $1+x^{2}+y^{2}$ is a weighted function. We denote the subspace of all continuous functions belonging to $B_{\rho}$ by $C_{\rho}$. Let $C_{\rho}^{*}$ be the subspace of all functions $f \in C_{\rho}$, for which $\lim _{\sqrt{x^{2}+y^{2}} \longrightarrow \infty}(f(x, y) / \rho(x, y))$ is finite. The norm on $C_{\rho}^{*}$ is $\|f\|_{\rho}=\sup _{x, y \in I}(|f(x, y)| / \rho(x, y))$. For the infinite interval $I$, $f \in C_{\rho}^{*}$, and $\delta_{1}, \delta_{2}>0$, Ilarslan and Acar [34] introduced the weighted modulus of continuity as

$$
\begin{aligned}
& \Omega_{\rho}\left(f ; \delta_{1}, \delta_{2}\right) \\
& =\sup _{x, y \in I} \sup _{0 \leq\left|k_{1}\right| \leq \delta_{1}, 0 \leq\left|k_{2}\right| \leq \delta_{2}} \frac{\left|f\left(x+k_{1}, y+k_{2}\right)-f(x, y)\right|}{\rho(x, y) \rho\left(k_{1}, k_{2}\right)},
\end{aligned}
$$

which satisfies the following inequality:

$$
\begin{array}{r}
\Omega_{\rho}\left(f ; d_{1} \delta_{1}, d_{2} \delta_{2}\right) \leq 4\left(1+d_{1}\right)\left(1+d_{2}\right)\left(1+\delta_{1}^{2}\right) \\
\cdot\left(1+\delta_{2}^{2}\right) \Omega_{\rho}\left(f ; \delta_{1}, \delta_{2}\right), \\
d_{1}, d_{2}>0 .
\end{array}
$$

From the definition of $\Omega_{\rho}$, we have

$$
\begin{aligned}
& |f(t, s)-f(x, y)| \leq \rho(x, y) \rho(|t-x|,|s-y|) \\
& \cdot \Omega_{\rho}(f ;|t-x|,|s-y|) \leq\left(1+x^{2}+y^{2}\right) \\
& \cdot\left(1+(t-x)^{2}\right)\left(1+(s-y)^{2}\right) \\
& \cdot \Omega_{\rho}(f ;|t-x|,|s-y|) .
\end{aligned}
$$

Now, we establish the degree approximation of operators $C_{p_{n_{1}}, p_{n_{2}}, q_{n_{1}}, q_{n_{2}}}^{n_{1}}$ in the weighted space $C_{\rho}^{*}$ by the weighted modulus of continuity $\Omega_{\rho}$.

Theorem 19. For $f \in C_{\rho}^{*}$, we have the following inequality:

$$
\begin{gathered}
\sup _{x, y \in I} \frac{C_{p_{n_{1}}, p_{n_{2}}, q_{n_{1}}, q_{n_{2}}}^{n_{n_{2}}, \alpha, l}(f ; x, y)-f(x, y)}{(\rho(x, y))^{3}} \\
\leq C \Omega_{\rho}\left(f ; \frac{b_{n_{1}}}{\sqrt{\left[n_{1}\right]_{p_{n_{1}}, q_{n_{1}}}}}, \frac{b_{n_{2}}}{\sqrt{\left[n_{2}\right]_{p_{n_{2}}, q_{n_{2}}}}}\right),
\end{gathered}
$$

where $C$ is a positive constant.

Proof. From (81) and (82), for $\delta_{n_{1}}, \delta_{n_{2}}>0$, we get

$$
\begin{aligned}
& |f(t, s)-f(x, y)|=4\left(1+x^{2}+y^{2}\right)\left(1+(t-x)^{2}\right) \\
& \cdot\left(1+(s-y)^{2}\right)\left(1+\frac{|t-x|}{\delta_{n_{1}}}\right)\left(1+\frac{|s-y|}{\delta_{n_{2}}}\right) \\
& \cdot\left(1+\delta_{n_{1}}^{2}\right) \times\left(1+\delta_{n_{2}}^{2}\right) \Omega_{\rho}\left(f ; \delta_{n_{1}}, \delta_{n_{2}}\right) \\
& =4\left(1+x^{2}+y^{2}\right)\left(1+\delta_{n_{1}}^{2}\right)\left(1+\delta_{n_{2}}^{2}\right) \\
& \cdot\left(1+\frac{|t-x|}{\delta_{n_{1}}}+(t-x)^{2}+\frac{|t-x|}{\delta_{n_{1}}}(t-x)^{2}\right) \\
& \quad \times\left(1+\frac{|s-y|}{\delta_{n_{2}}}+(s-y)^{2}+\frac{|s-y|}{\delta_{n_{2}}}(s-y)^{2}\right) \\
& \cdot \Omega_{\rho}\left(f ; \delta_{n_{1}}, \delta_{n_{2}}\right),
\end{aligned}
$$

and, applying the operators $C_{p_{n_{1}}, p_{n_{2}}, q_{n_{1}}, q_{n_{2}}}^{n_{n_{2}}}$ on the above inequality, we have

$$
\begin{aligned}
& \left|C_{p_{n_{1}}, p_{n_{2}}, q_{n_{1}}, q_{n_{2}}}^{n_{1}, \widetilde{n_{2}, \alpha, l}}(f ; x, y)-f(x, y)\right| \\
& \quad \leq C_{p_{n_{1}}, p_{n_{2}}, q_{n_{1}}, q_{n_{2}}}^{n_{1}, \widetilde{n_{2}, \alpha, \beta, l}}(|f(t, s)-f(x, y)| ; x, y) \leq 4(1 \\
& \left.\quad+x^{2}+y^{2}\right)\left(1+\delta_{n_{1}}^{2}\right)\left(1+\delta_{n_{2}}^{2}\right) C_{p_{n_{1}}, p_{n_{2}}, q_{n_{1}}, q_{n_{2}}}^{n_{1}, \widetilde{n_{2}, \alpha, \beta, l}}(1
\end{aligned}
$$




$$
\begin{aligned}
& \left.+\frac{|t-x|}{\delta_{n_{1}}}+(t-x)^{2}+\frac{|t-x|}{\delta_{n_{1}}}(t-x)^{2} ; x, y\right) \\
& \cdot C_{p_{n_{1}}, p_{n_{2}}, q_{n_{1}}, q_{n_{2}}}^{n_{1}, n_{2}, \beta} \\
& \left.+\frac{|s-y|}{\delta_{n_{2}}}(s-y)^{2} ; x, y\right) \times \Omega_{\rho}\left(f ; \delta_{n_{1}}, \delta_{n_{2}}\right)=4(1 \\
& \left.+x^{2}+y^{2}\right)\left(1+\delta_{n_{1}}^{2}\right)\left(1+\delta_{n_{2}}^{2}\right)(1 \\
& +\frac{C_{p_{n_{1}}, p_{n_{2}}, q_{n_{1}}, q_{n_{2}}}^{n_{n_{1}}, \alpha, \beta}(|t-x| ; x, y)}{\delta_{n_{1}}} \\
& +C_{p_{n_{1}}, p_{n_{2}}, q_{n_{1}}, q_{n_{2}}}^{n_{1}, n_{2}, \beta_{2}, l}\left((t-x)^{2} ; x, y\right)
\end{aligned}
$$

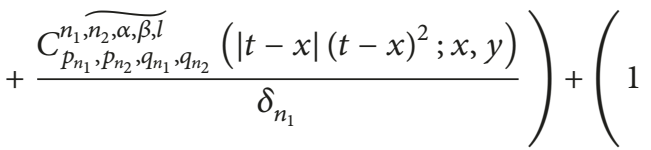

$$
\begin{aligned}
& +\frac{C_{p_{n_{1}}, p_{n_{2}}, q_{n_{1}}, q_{n_{2}}}^{\left.n_{1}, \alpha-y \mid ; x, y\right)}}{\delta_{n_{2}}} \\
& +C_{p_{n_{1}}, p_{n_{2}}, q_{n_{1}}, q_{n_{2}}}^{n_{1}, n_{2}, \alpha, \beta}\left((s-y)^{2} ; x, y\right) \\
& \left.+\frac{C_{p_{n_{1}}, p_{n_{2}}, q_{n_{1}}, q_{n_{2}}}^{\left.n_{1}, \alpha-y \mid(s-y)^{2} ; x, y\right)}}{\delta_{n_{2}}}\right) \\
& \cdot \Omega_{\rho}\left(f ; \delta_{n_{1}}, \delta_{n_{2}}\right) .
\end{aligned}
$$

Using Cauchy-Schwarz inequality, we get

$\left|C_{P_{n_{1}}, p_{n_{2}}, q_{n_{1}}, q_{n_{2}}}^{n_{1}, n_{2}, \beta},(f x, y)-f(x, y)\right| \leq 4\left(1+x^{2}+y^{2}\right)$

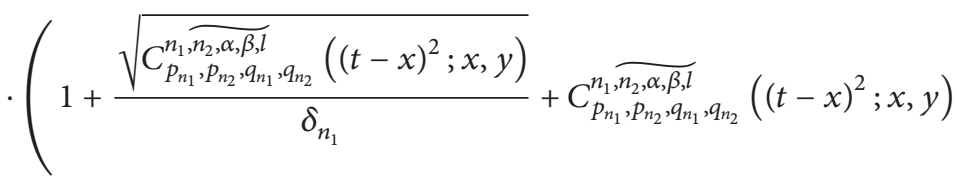

$$
\begin{aligned}
& \left.+\frac{\sqrt{\left.C_{p_{n_{1}}, p_{n_{2}}, q_{n_{1}}, q_{n_{2}}}^{n_{1}, \alpha, \beta},(t-x)^{2} ; x, y\right)} \sqrt{C_{p_{n_{1}}, p_{n_{2}}, q_{n_{1}}, q_{n_{2}}}^{n_{1}, \widetilde{n_{2}, \alpha, l}}\left((t-x)^{4} ; x, y\right)}}{\delta_{n_{1}}}\right) \times\left(1+\delta_{n_{1}}^{2}\right) \\
& \cdot\left(1+\delta_{n_{2}}^{2}\right)\left(1+\frac{\sqrt{C_{p_{n_{1}}, p_{n_{2}}, q_{n_{1}}, q_{n_{2}}, \eta_{2}, \beta}^{n_{2}}\left((s-y)^{2} ; x, y\right)}}{\delta_{n_{2}}}+C_{p_{n_{1}}, p_{n_{2}}, q_{n_{1}}, q_{n_{2}}}^{n_{1}, n_{1}, \alpha, l}(s-y)^{2} ; x, y\right) \\
& \left.+\sqrt{C_{p_{n_{1}}, p_{n_{2}}, q_{n_{1}}, q_{n_{2}}}^{n_{1}, \widetilde{n_{2}, \alpha, l}}\left((s-y)^{2} ; x, y\right)} \times \frac{\sqrt{C_{p_{n_{1}}, p_{n_{2}}, q_{n_{1}}, q_{n_{2}}, q_{2}, q_{2}, l}\left((s-y)^{4} ; x, y\right)}}{\delta_{n_{2}}}\right) \\
& \text { - } \Omega_{\rho}\left(f ; \delta_{n_{1}}, \delta_{n_{2}}\right) .
\end{aligned}
$$

Using Lemma 18, we have

$$
\begin{aligned}
& \cdot \sqrt{O\left(\frac{b_{n_{1}}}{\left[n_{1}\right]_{p_{n_{1}}, q_{n_{1}}}}\right) x}+O\left(\frac{b_{n_{1}}}{\left[n_{1}\right]_{p_{n_{1}}, q_{n_{1}}}}\right) x+\frac{1}{\delta_{n_{1}}} \\
& \left.\cdot \sqrt{O\left(\frac{b_{n_{1}}}{\left[n_{1}\right]_{p_{n_{1}}, q_{n_{1}}}}\right)} x \sqrt{O\left(\frac{b_{n_{1}}^{2}}{\left[n_{1}\right]_{p_{n_{1}}, q_{n_{1}}}^{2}}\right) x^{2}}\right] \\
& \times\left[1+\frac{1}{\delta_{n_{2}}} \sqrt{O\left(\frac{b_{n_{2}}}{\left[n_{2}\right]_{p_{n_{2}}, q_{n_{2}}}}\right) y}\right.
\end{aligned}
$$$$
\left|C_{p_{n_{1}}, p_{n_{2}}, q_{n_{1}}, q_{n_{2}}}^{n_{1}, n_{2}, \beta}(f ; x, y)-f(x, y)\right| \leq 4\left(1+x^{2}+y^{2}\right)
$$$$
\cdot\left(1+\delta_{n_{1}}^{2}\right)\left(1+\delta_{n_{2}}^{2}\right)\left[1+\frac{1}{\delta_{n_{1}}}\right.
$$ 
TABLE 3: The absolute error bound of the approximation of $\left.C_{p_{n_{1}}, p_{n_{2}}, q_{n_{1}}, q_{n_{2}}}^{n_{2}, \alpha}, f ; x, y\right)$ with $\alpha=1, \beta=2, l=5, b_{n_{1}}=b_{n_{2}}=10$ and different values of $n_{1}=n_{2}=n, p_{n_{1}}=p_{n_{2}}=p, q_{n_{1}}=q_{n_{2}}=q$, where $p=1-1 / 10^{m}, q=1-1 / 10^{m-1}$.

\begin{tabular}{lcccc}
\hline$m$ & & $\left\|f(x, y)-C_{p_{n_{1}}, p_{n_{2}}, q_{n_{1}}, q_{n_{2}}}^{n_{1}, \alpha_{2}}(f ; x, y)\right\|_{\infty}$ & $n=80$ & $n=120$ \\
\hline 6 & $n=30$ & $n=50$ & 0.20568442 & 0.14177371 \\
7 & 0.44324007 & 0.30755815 & 0.20562961 & 0.14171254 \\
8 & 0.44321848 & 0.30751521 & 0.20562413 & 0.14170642 \\
9 & 0.44321632 & 0.30751092 & 0.20562358 & 0.14170581 \\
10 & 0.44321610 & 0.30751049 & 0.20562352 & 0.14170575 \\
\hline
\end{tabular}

$$
\begin{aligned}
& +O\left(\frac{b_{n_{2}}^{2}}{\left[n_{2}\right]_{p_{n_{2}}, q_{n_{2}}}^{2}}\right) y+\frac{1}{\delta_{n_{2}}} \\
& \left.\cdot \sqrt{O\left(\frac{b_{n_{2}}}{\left[n_{2}\right]_{p_{n_{2}}, q_{n_{2}}}^{2}}\right) y} \sqrt{O\left(\frac{b_{n_{2}}^{2}}{\left[n_{2}\right]_{p_{n_{2}}, q_{n_{2}}}^{2}}\right) y^{2}}\right] \\
& \cdot \Omega_{\rho}\left(f ; \delta_{n_{1}}, \delta_{n_{2}}\right) .
\end{aligned}
$$

Then, we have

$$
\begin{aligned}
& \left|C_{p_{n_{1}}, p_{n_{2}}, q_{n_{1}}, q_{n_{2}}}^{n_{1}, n_{n_{1}}, \beta, l}(f ; x, y)-f(x, y)\right| \leq 4\left(1+x^{2}+y^{2}\right) \\
& \cdot\left(1+\delta_{n_{1}}^{2}\right)\left(1+\delta_{n_{2}}^{2}\right)\left(1+\frac{1}{\delta_{n_{1}}} \sqrt{\frac{C_{1} b_{n_{1}}}{\left[n_{1}\right]_{p_{n_{1}}, q_{n_{1}}}}} x^{1 / 2}\right. \\
& \left.+\frac{C_{1} b_{n_{1}}}{\left[n_{1}\right]_{p_{n_{1}}, q_{n_{1}}}} x+\frac{1}{\delta_{n_{1}}} \sqrt{\frac{C_{1} C_{2} b_{n_{1}}^{3}}{\left[n_{1}\right]_{p_{n_{1}}, q_{n_{1}}}^{3}} x^{3 / 2}}\right)(1 \\
& +\frac{1}{\delta_{n_{2}}} \sqrt{\frac{C_{3} b_{n_{2}}}{\left[n_{2}\right]_{p_{n_{2}}, q_{n_{2}}}}} y^{1 / 2}+\frac{C_{3} b_{n_{2}}}{\left[n_{2}\right]_{p_{n_{2}}, q_{n_{2}}}} y \\
& \left.+\frac{1}{\delta_{n_{2}}} \sqrt{\frac{C_{3} C_{4} b_{n_{2}}^{3}}{\left[n_{2}\right]_{p_{n_{2}}, q_{n_{2}}}^{3}}} y^{3 / 2}\right) \Omega_{\rho}\left(f ; \delta_{n_{1}}, \delta_{n_{2}}\right) .
\end{aligned}
$$

Let $\delta_{n_{1}}=\sqrt{b_{n_{1}} /\left[n_{1}\right]_{p_{n_{1}}, q_{n_{1}}}}$ and $\delta_{n_{2}}=\sqrt{b_{n_{2}} /\left[n_{2}\right]_{p_{n_{2}}, q_{n_{2}}}}$; we have

$$
\begin{gathered}
\left|C_{p_{n_{1}}, p_{n_{2}}, q_{n_{1}}, q_{n_{2}}}^{n_{1}, n_{2}, \beta, l}(f ; x, y)-f(x, y)\right| \leq 4\left(1+x^{2}+y^{2}\right) \\
\quad\left(1+\frac{b_{n_{1}}}{\left[n_{1}\right]_{p_{n_{1}}, q_{n_{1}}}}\right)\left(1+\frac{b_{n_{2}}}{\left[n_{2}\right]_{p_{n_{2}}, q_{n_{2}}}}\right)
\end{gathered}
$$

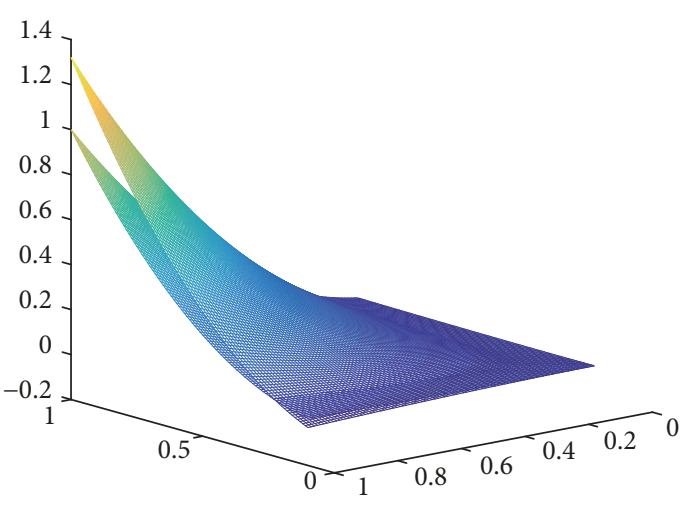

FIGURE 5: The figures of (the upper one) $C_{p_{n_{1}}, p_{n_{2}}, q_{n_{1}}, q_{n_{2}}}^{n_{2}, \alpha, l}(f ; x, y)$ with $n_{1}=n_{2}=50, p_{n_{1}}=p_{n_{2}}=0.999, q_{n_{1}}=q_{n_{2}}=0.95, \alpha=1, \beta=2, l=$ $5, b_{n_{1}}=b_{n_{2}}=5$ and $f(x, y)$ (the below one).

$$
\begin{aligned}
& \cdot C\left(1+x^{2}+y^{2}\right)^{2} \\
& \times \Omega_{\rho}\left(f ; \sqrt{\frac{b_{n_{1}}}{\left[n_{1}\right]_{p_{n_{1}}, q_{n_{1}}}}}, \sqrt{\frac{b_{n_{2}}}{\left[n_{2}\right]_{p_{n_{2}}, q_{n_{2}}}}}\right),
\end{aligned}
$$

where $C, C_{1}, C_{2}, C_{3}, C_{4}$ are positive constants. Theorem 19 is proved.

\section{Graphical and Numerical Analysis II}

In this section, we give several graphs and numerical examples to show the convergence of $C_{p_{n_{1}}, p_{n_{2}}, q_{n_{1}}, q_{n_{2}}}^{n_{n_{2}, \alpha, \beta,}}(f ; x, y)$ to $f(x, y)$ with different values of parameters which satisfy the conclusions of Lemmas 4 and 5.

Example 20. Let $f(x, y)=x^{2} y^{2}$; the graphs of $C_{p_{n_{1}}, p_{n_{2}}, q_{n_{1}}, q_{n_{2}}}^{n_{1}, n_{2}, \beta}(f ; x, y)$ with $n_{1}=n_{2}=50, p_{n_{1}}=p_{n_{2}}=$ $0.999, q_{n_{1}}=q_{n_{2}}=0.95, \alpha=1, \beta=2, l=5, b_{n_{1}}=b_{n_{2}}=5$, and $f(x, y)$ are shown in Figure 5. The graphs of $C_{p_{n_{1}}, p_{n_{2}}, q_{n_{1}}, q_{n_{2}}}^{n_{1}, n_{n_{2}}, \beta}(f ; x, y)$ with $n_{1}=n_{2}=100, p_{n_{1}}=p_{n_{2}}=$ $0.99999, q_{n_{1}}=q_{n_{2}}=0.999, \alpha=1, \beta=2, l=5, b_{n_{1}}=b_{n_{2}}=10$, and $f(x, y)$ are shown in Figure 6. In Table 3 , we show the absolute error bound of the approximation of 


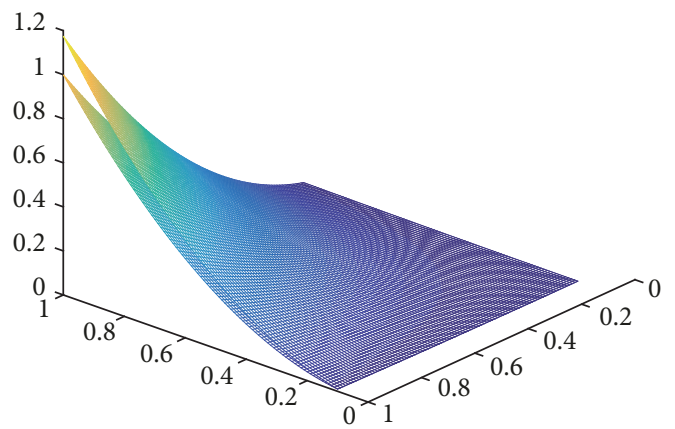

FIGURE 6: The figures of (the upper one) $C_{p_{n_{1}}, p_{n_{2}}, q_{n_{1}}, q_{n_{2}}}^{n_{n_{2}, \alpha, \beta}}(f ; x, y)$ with $n_{1}=n_{2}=100, p_{n_{1}}=p_{n_{2}}=0.99999, q_{n_{1}}=q_{n_{2}}=0.999, \alpha=1, \beta=$ $2, l=5, b_{n_{1}}=b_{n_{2}}=10$ and $f(x, y)$ (the below one).

$C_{p_{n_{1}}, n_{n_{2}}, q_{n_{1}}, q_{n_{2}}}^{n_{1}, \alpha, \beta}(f ; y)$ with $\alpha=1, \beta=2, l=5, b_{n_{1}}=b_{n_{2}}=10$ and different values of $n_{1}=n_{2}=n, p_{n_{1}}=p_{n_{2}}=p, q_{n_{1}}=q_{n_{2}}=$ $q$, where $p=1-1 / 10^{m}$ and $q=1-1 / 10^{m-1}(m=6,7,8,9,10)$, to $f(x, y)$.

\section{Data Availability}

The data used to support the findings of this study are included within the article.

\section{Conflicts of Interest}

The authors declare that they have no conflicts of interest.

\section{Acknowledgments}

This work is supported by the National Natural Science Foundation of China (Grant no. 11601266), the Natural Science Foundation of Fujian Province of China (Grant no. 2016J05017), and the Program for New Century Excellent Talents in Fujian Province University. The authors also thank Fujian Provincial Key Laboratory of Data Intensive Computing and Key Laboratory of Intelligent Computing and Information Processing of Fujian Province University.

\section{References}

[1] S. N. Bernstein, "Démonstration du théorème de Weierstrass fondée sur la calcul des probabilités," Comm. Soc. Math. Charkow Sér, vol. 13, pp. 1-2, 1912.

[2] S. A. Mohiuddine, T. Acar, and A. Alotaibi, "Construction of a new family of Bernstein-Kantorovich operators," Mathematical Methods in the Applied Sciences, vol. 40, no. 3, pp. 7749-7759, 2017.

[3] T. Acar and A. Kajla, "Degree of Approximation for Bivariate Generalized Bernstein Type Operators," Results in Mathematics, vol. 79, no. 2, 2018.

[4] S. A. Mohiuddine, T. Acar, and M. Alghamdi, "Genuine modified Bernstein-Durrmeyer operators," Journal of Inequalities and Applications, vol. 104, 2018.
[5] Q.-B. Cai, B.-Y. Lian, and G. Zhou, "Approximation properties of $\lambda$-Bernstein operators," Journal of Inequalities and Applications, vol. 61, 2018.

[6] A. Lupas, "A q-analogue of the Bernstein operator," in Proceedings of the Seminar on Numerical and Statistical Calculus, pp. 85-92, University of Cluj-Napoca, 1987.

[7] G. M. Phillips, "Bernstein polynomials based on the $q$-integers," Annals of Numerical Mathematics, vol. 4, no. 1-4, pp. 511-518, 1997.

[8] M. Mursaleen, K. J. Ansari, and A. Khan, “On ( $p, q)$-analogue of Bernstein operators," Applied Mathematics and Computation, vol. 266, pp. 874-882, 2015.

[9] M. Mursaleen, K. J. Ansari, and A. Khan, "Some approximation results by (p, q)-analogue of Bernstein-Stancu operators," Applied Mathematics and Computation, vol. 264, pp. 392-402, 2015.

[10] S. M. Kang, A. Rafiq, A.-M. Acu, F. Ali, and Y. C. Kwun, "Some approximation properties of ( $\mathrm{p}, \mathrm{q}$ )-Bernstein operators," Journal of Inequalities and Applications, vol. 169, 2016.

[11] A. Khan and V. Sharma, "Statistical approximation by (p, q)analogue of Bernstein-Stancu operators," 2017.

[12] T. Acar, A. Aral, and S. A. Mohiuddine, "Approximation by Bivariate (p, q)-Bernstein-Kantorovich Operators," Iranian Journal of Science \& Technology, vol. 42, no. 2, pp. 655-662, 2018.

[13] M. Mursaleen, F. Khan, and A. Khan, "Approximation by (p, q)Lorentz polynomials on a compact disk," Complex Analysis and Operator Theory, vol. 10, no. 8, pp. 1725-1740, 2016.

[14] M. Mursaleen, M. Nasiruzzaman, A. Khan, and K. J. Ansari, "Some approximation results on Bleimann-Butzer-Hahn operators defined by (p, q)-integers," Filomat, vol. 30, no. 3, pp. 639648, 2016.

[15] M. Mursaleen and A. Khan, "Generalized q-Bernstein-Schurer operators and some approximation theorems," Journal of Function Spaces and Applications, vol. 2013, Article ID 719834, 7 pages, 2013.

[16] U. Kadak, "On weighted statistical convergence based on (p, q)integers and related approximation theorems for functions of two variables," Journal of Mathematical Analysis and Applications, vol. 443, no. 2, pp. 752-764, 2016.

[17] T. Acar, P. N. Agrawal, and A. Sathish Kumar, "On a modification of (p, q)-Szász-Mirakyan operators," Complex Analysis and Operator Theory, vol. 12, no. 1, pp. 1-3, 2018.

[18] T. Acar, A. Aral, and S. A. Mohiuddine, "On Kantorovich modification of (p, q)-Baskakov operators," Journal of Inequalities and Applications, vol. 98, 2016.

[19] A. D. Gadjiev, R. O. Efendiev, and E. Ibikli, "Generalized Bernstein-Chlodowsky polynomials," Rocky Mountain Journal of Mathematics, vol. 28, no. 4, pp. 1267-1277, 1998.

[20] E. A. Gadjieva and E. Ibikli, "Weighted approximation by Bernstein-Chlodowsky polynomials," Indian Journal of Pure and Applied Mathematics, vol. 30, no. 1, pp. 83-87, 1999.

[21] H. Karsli and V. Gupta, "Some approximation properties of qChlodowsky operators," Applied Mathematics and Computation, vol. 195, no. 1, pp. 220-229, 2008.

[22] A. E. Piriyeva, "On order of approximation of functions by generalized Bernstein-Chlodowsky polynomials," Proceedings of Institute of Mathematics and Mechanics. National Academy of Sciences of Azerbaijan, vol. 21, pp. 157-164, 2004.

[23] I. Büyükyazici and E. Ibikli, "Inverse theorems for BernsteinChlodowsky type polynomials," Journal of Mathematics of Kyoto University, vol. 46, pp. 21-29, 2006. 
[24] E. A. Gadjieva and T. K. Gasanova, "Approximation by two dimensional Bernstein-Chlodowsky polynomials in triangle with mobile boundary," Transactions of Azerbaijan National Academy of Sciences. Series of Physical-Technical and Mathematical Sciences, vol. 20, no. 4, Math. Mech., pp. 47-51, 265, 2000.

[25] E. Ibikli, "On approximation for functions of two variables on a triangular domain," Rocky Mountain Journal of Mathematics, vol. 35, no. 5, pp. 1523-1531, 2005.

[26] V. N. Mishra, M. Mursaleen, S. Pandey, and A. Alotaibi, "Approximation properties of Chlodowsky variant of ( $p, q)$ Bernstein-Stancu-SCHurer operators," Journal of Inequalities and Applications, vol. 176, 2017.

[27] M. N. Hounkonnou, J. Désiré, and B. Kyemba, "R(p, q)-calculus: differentiation and integration," SUT Journal of Mathematics, vol. 49, pp. 145-167, 2013.

[28] R. Jagannathan and K. S. Rao, "Two-parameter quantum algebras, twin-basic numbers, and associated generalized hypergeometric series," in Proceedings of the International Conference on Number Theory and Mathematical Physics, pp. 20-21, 2005.

[29] J. Katriel and M. Kibler, "Normal ordering for deformed boson operators and operator-valued deformed Stirling numbers," Journal of Physics A: Mathematical and General, vol. 24, no. 9, pp. 2683-2691, 1992.

[30] P. N. Sadjang, "On the fundamental theorem of (p, q)-calculus and some (p, q)-Taylor formulas," 2015.

[31] V. Sahai and S. Yadav, "Representations of two parameter quantum algebras and p, q-special functions," Journal of Mathematical Analysis and Applications, vol. 335, no. 1, pp. 268-279, 2007.

[32] Q.-B. Cai and X.-W. Xu, "A basic problem of (p, q)-Bernstein operators," Journal of Inequalities and Applications, vol. 140, 2017.

[33] R. A. DeVore and G. G. Lorentz, Constructive Approximation, vol. 303, Springer, Berlin, Germany, 1993.

[34] H. G. Ilarslan and T. Acar, "Approximation by bivariate (p,q)-Baskakov-Kantorovich operators," Georgian Mathematical Journal, 2016. 


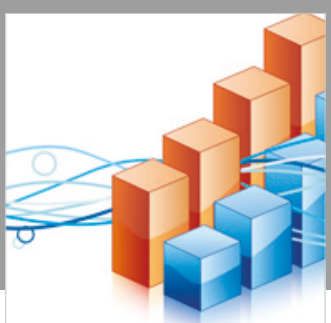

Advances in

Operations Research

\section{-n-m}
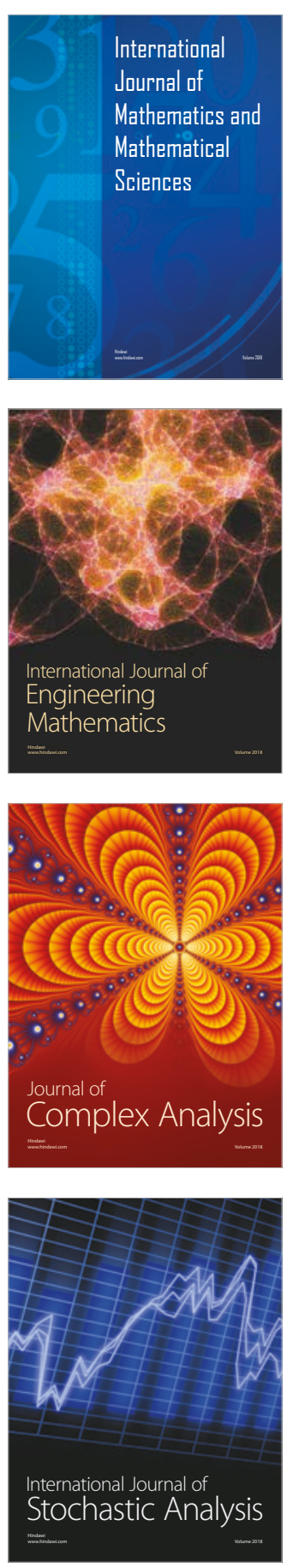
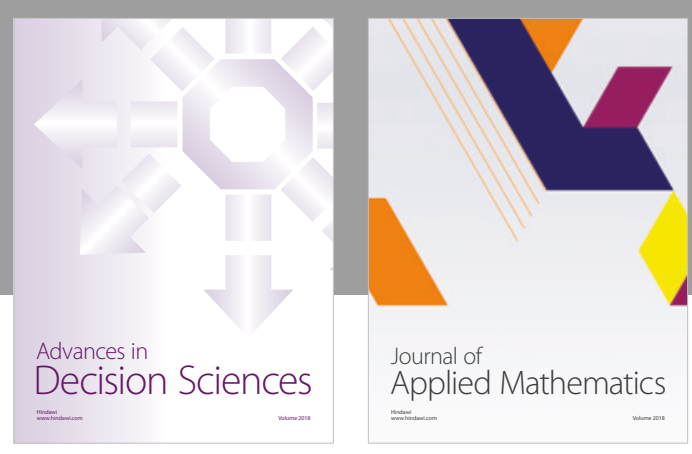

Journal of

Applied Mathematics
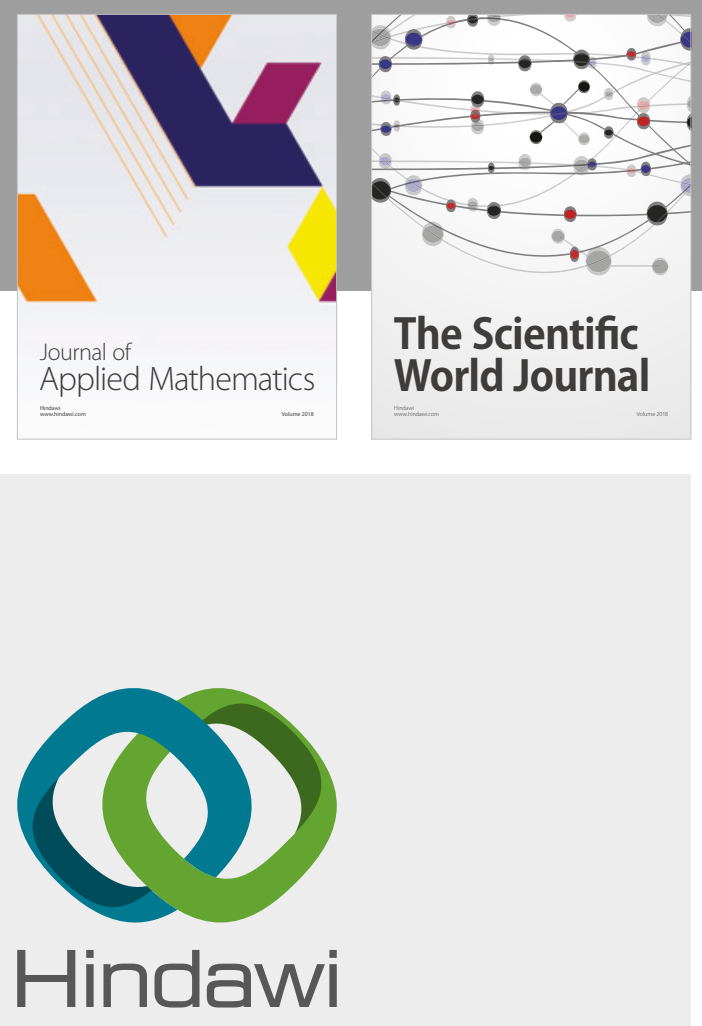

Submit your manuscripts at

www.hindawi.com

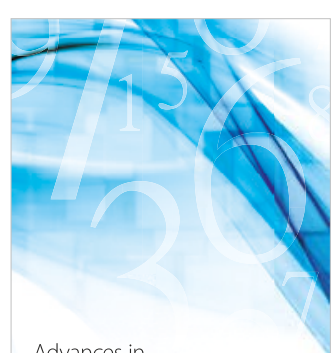

Advances in
Numerical Analysis
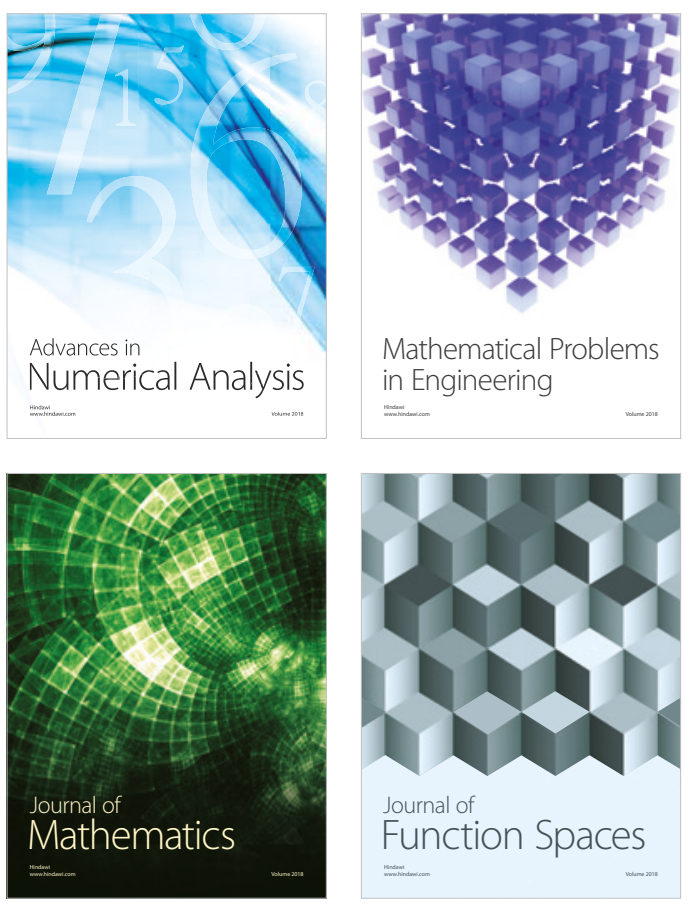

Mathematical Problems in Engineering

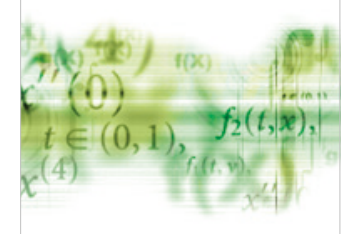

International Journal of

Differential Equations

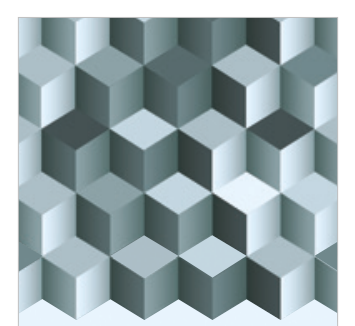

Journal of

Function Spaces

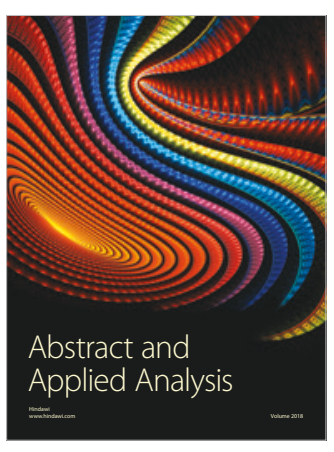

The Scientific

World Journal

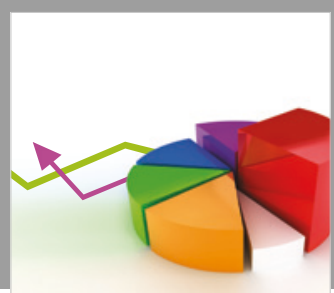

Journal of

Probability and Statistics
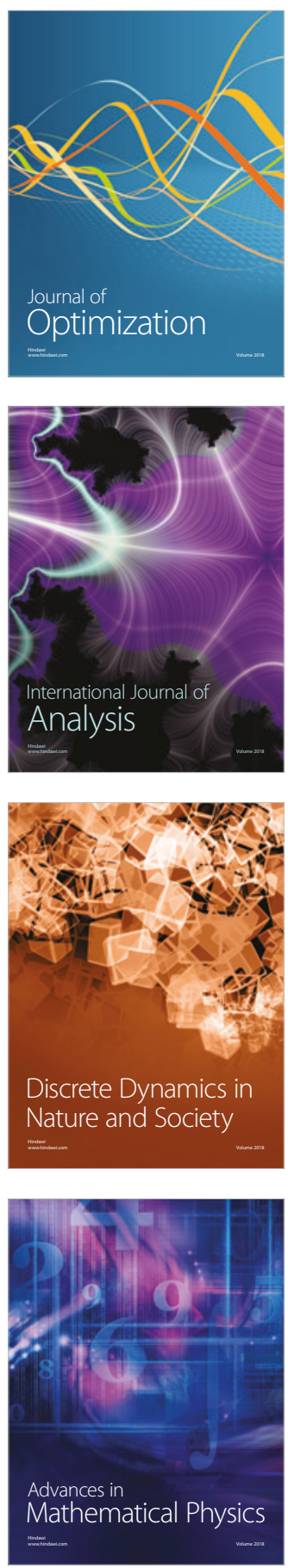